\title{
AG01 regulates pericentromeric regions in mouse embryonic stem cells
}

\author{
Madlen Müller ${ }^{1,2} \mathbb{D}$, Tara Fäh¹, Moritz Schaefer ${ }^{1,2}$ (D), Victoria Hermes ${ }^{1}$, Janina Luitz ${ }^{1}$, Patrick Stalder ${ }^{1,2}$, Rajika Arora $\mathbb{D}^{1}$, \\ Richard Patryk Ngondo ${ }^{1}$, Constance Ciaudo ${ }^{1}$ (D)
}

\begin{abstract}
Argonaute proteins (AGOs), which play an essential role in cytosolic post-transcriptional gene silencing, have been also reported to function in nuclear processes like transcriptional activation or repression, alternative splicing and, chromatin organization. As most of these studies have been conducted in human cancer cell lines, the relevance of AGOs nuclear functions in the context of mouse early embryonic development remains uninvestigated. Here, we examined a possible role of the AGO1 protein on the distribution of constitutive heterochromatin in mouse embryonic stem cells (mESCs). We observed a specific redistribution of the repressive histone mark $\mathrm{H} 3 \mathrm{~K} 9 \mathrm{me} 3$ and the heterochromatin protein HP1 $\alpha$, away from pericentromeric regions upon Ago1 depletion. Furthermore, we demonstrated that major satellite transcripts are strongly up-regulated in Ago1_KO mESCs and that their levels are partially restored upon AG01 rescue. We also observed a similar redistribution of $\mathrm{H} 3 \mathrm{~K} 9 \mathrm{me} 3$ and HP1 $\alpha$ in Drosha_KO mESCs, suggesting a role for microRNAs (miRNAs) in the regulation of heterochromatin distribution in mESCs. Finally, we showed that specific miRNAs with complementarity to major satellites can partially regulate the expression of these transcripts.
\end{abstract}

DOI 10.26508/Isa.202101277 | Received 25 October 2021 | Revised 17 February 2022 | Accepted 17 February 2022 | Published online 2 March 2022

\section{Introduction}

The miRNA pathway is crucial in regulating early embryonic development and differentiation in vivo and in vitro (DeVeale et al, 2021). MiRNAs can fine-tune gene expression throughout early embryonic development at the post-transcriptional level. MiRNA precursors are processed into $\sim 22$-nt long mature miRNAs by two consecutive cleavage steps conducted by the RNAse III enzyme DROSHA in the nucleus, and DICER, in the cytoplasm (Bodak et al, 2017). Mature miRNAs are loaded into Argonaute (AGO) proteins, which are key components of the RNA-induced silencing complex.
They guide the RNA-induced silencing complex to partially complementary target sequences leading to the translational inhibition of these targets (Bartel, 2018).

In mice, there are four AGO proteins (AGO1-4), but only AGO1 and AGO2 are detectably expressed during early embryonic development, with $\mathrm{AGO} 2$ being substantially more abundant (Lykke-Andersen et al, 2008; Boroviak et al, 2018; Müller et al, 2020). Whereas Ago2deficient mice die at a post-implantation stage, because of severe developmental defects (Liu et al, 2004; Alisch et al, 2007; Morita et al, 2007; Cheloufi et al, 2010), Ago1,3,4-deficient mice are viable (Modzelewski et al, 2012; Van Stry et al, 2012).

Mouse embryonic stem cells (mESCs), which are derived from the inner cell mass of the blastocyst, are a powerful tool to study early embryonic development in vitro. These cells are pluripotent and can differentiate into the three embryonic germ layers. As observed in vivo, mESCs only express AGO1 and AGO2 proteins (LykkeAndersen et al, 2008; Boroviak et al, 2018; Müller et al, 2020). MESCs deficient for either AGO1 or AGO2 are viable, can exit from pluripotency and differentiate into the three embryonic germ layers (Ngondo et al, 2018).

In addition to their major role in the cytoplasmic miRNA pathway, several studies have reported noncanonical functions of the AGO proteins in the nucleus (Meister, 2013; Gagnon et al, 2014a; Li et al, 2020). AGO2 was shown to shuttle into the nucleus with the help of TNRC6A (Nishi et al, 2013). In the nucleus, guided by small RNAs (smRNAs), both AGO1 and AGO2 have been shown to localize to promoter regions and reinforce the recruitment of chromatin modifiers leading to either transcriptional activation or silencing (Janowski et al, 2006; Kim et al, 2006; Li et al, 2006; Hu et al, 2012; Cho et al, 2014; Portnoy et al, 2016). AG01 was also found to be enriched at promoters of actively transcribed genes, where it interacts with RNA Polymerase II (RNA PolII) (Huang et al, 2013). Furthermore, AGO1 was found to localize to enhancer regions, which was dependent on a species of RNA called enhancer RNAs (Alló et al, 2014; Shuaib et al, 2019). In addition, interaction of AGO1 with enhancers was shown to be crucial for maintenance of 3D chromatin organization and more recently to control myogenic differentiation (Shuaib et al, 2019;

${ }^{1}$ Swiss Federal Institute of Technology Zurich, Institute of Molecular Health Sciences (IMHS), Chair of RNAi and Genome Integrity, Zurich, Switzerland ${ }^{2}$ Life Science Zurich Graduate School, University of Zürich, Zürich, Switzerland 
Fallatah et al, 2021). Finally, AGO1 has been implicated in alternative splicing events, taking place within the nucleus (Ameyar-Zazoua et al, 2012; Alló et al, 2014; Agirre et al, 2015). Of note, most of these chromatin-associated functions have been described mainly for AGO1, whereas AGO2 was reported to be involved in double-strand break repair (Gao et al, 2014; Wang \& Goldstein, 2016).

Most of the aforementioned AGOs functions were described in human cancer cell lines and have not been studied during early embryonic development. Only few studies reported phenotypes associating the AGO proteins with other functions in mESCS (Sarshad et al, 2018; Kelly et al, 2019; Shivram et al, 2019). For instance, Kelly et al (2019) identified that TGF- $\beta$ pathway targets are up-regulated upon $\mathrm{AGO} 2$ depletion in $\mathrm{mESCS}$, due to a lack of miRNA repression. The up-regulation of these targets additionally correlated with decreased levels of the repressive histone mark, H3K27me3 (Kelly et al, 2019). Interestingly, we also observed a specific loss of H3K27me3 mark in Ago1\&2_KO mESCs (Mueller et al, 2021 Preprint). Repressive histone marks are important for the formation of heterochromatin, which is localized to distinct territories within the nucleus (Akhtar \& Gasser, 2007; Solovei et al, 2009).

In this study, we aimed to explore the link between AGO1 and heterochromatin by assessing both the amount and the distribution of constitutive heterochromatin in Ago1 mutant mESCs. We observed a specific redistribution of the repressive histone mark H3K9me3 and, the heterochromatin protein HP1 $\alpha$ away from pericentromeric regions in Ag01_KO mESCS. Furthermore, these regions are characterized by AT-rich tandem repeats known as major satellite sequences. We demonstrated that major satellite transcripts are strongly up-regulated in Ag01_KO mESCs. Nevertheless, we did not observe any changes in integrity of the pericentromeric region at the DNA level. Importantly, these phenotypes were rescued upon the reintroduction of AG01 in the mutant cells. These results prompted us to investigate the underlying molecular mechanism by which AGO1 might regulate major satellite transcripts. We first demonstrated the involvement of miRNAs by observing a similar redistribution of H3K9me3 and HP1 $\alpha$ in Drosha_KO mESCs. Using computational analyses and molecular approaches, we also found that AGO1, loaded with miR-30a, d, e-3p, might contribute partially to the regulation of major satellite transcripts. Overall, our results demonstrate for the first time a novel role for AGO1 in regulating major satellite transcripts and localization of H3K9me3 and HP1 $\alpha$ at pericentromeres in mESCS.

\section{Results}

\section{Ago1-depletion affects the distribution of H3K9me3 and HP1 $\alpha$ at pericentromeric regions}

Only the H3K27me3 heterochromatin mark, but not H3K9me3, was previously observed to be strongly down-regulated in Argonaute mutant mESCs (Kelly et al, 2019; Mueller et al, 2021 Preprint). Heterochromatin is localized to specific nuclear territories in mammalian cells (Akhtar \& Gasser, 2007; Solovei et al, 2009). H3K9me3, in particular, is enriched at pericentromeric constitutive heterochromatin regions in mammals, which can be found at the centromeres.
Constitutive heterochromatin at centromeres is required for proper sister chromatid cohesion and chromosome segregation (Bernard et al, 2001; Nonaka et al, 2002; Guenatri et al, 2004; Houlard et al, 2006; Probst \& Almouzni, 2008; Probst et al, 2009). Pericentromeric domains from several chromosomes are known to cluster together within interphase to form chromocenters (Guenatri et al, 2004; Probst \& Almouzni, 2008). Chromocenters are easily visible by fluorescence microscopy with a brighter DAPI stain (Guenatri et al, 2004; Probst \& Almouzni, 2008). AGO1 has been previously linked to chromatinassociated functions in mammalian cells (Huang et al, 2013; Alló et al, 2014; Shuaib et al, 2019). To study whether AGO1 might be important for constitutive heterochromatin localization in mESCs, we used two Ago1_KO mESC lines generated using a paired CRISPR-Cas9 approach (Wettstein et al, 2016). The first Ag01_KO1 mESC line was obtained from a previous study (Ngondo et al, 2018) and the second Ag01_KO2 mESCs line was newly generated and validated for the absence of AGO1 expression (Fig S1A and B). As previously observed in Ag01\&2_KO mESCS (Mueller et al, 2021 Preprint), the total amount of H3K9me3 histone mark as assessed by Western blotting (WB) was similar in WT versus Ago1_KO mESCs (Fig S1C). To go further, we analyzed the nuclear localization of H3K9me3 by indirect Immunofluorescence (IF) and observed colocalization of H3K9me3 with DAPIrich regions in WT MESCS (Figs $1 A$ and S1D). Surprisingly, this colocalization of H3K9me3 with DAPI-rich regions, was strongly reduced in Ago1_KO mESCs (Fig 1A).

To strengthen these observations, we performed H3K9me3 chromatin immunoprecipitation followed by quantitative PCR (ChIPqPCR) in WT versus Ago1_KO mESCS and successfully assessed the enrichment at known heterochromatic loci (Karimi et al, 2011; Ngondo et al, 2018) over a control region (Fig S1E and Table S1). We also compared the H3K9me3 enrichment at major satellites sequences in both WT and Ago1_KO, and observed that upon Ago1 depletion, around $50 \%$ of H3K9me3 is lost at these sites (Fig 1B and Table S1).

$\mathrm{H} 3 \mathrm{~K} 9 \mathrm{me} 3$ is deposited at pericentromeric heterochromatin regions by the methyltransferase SUV39H1/2, which is recruited there by HP1 $\alpha$ (also known as CBX5) (Bannister et al, 2001; Lachner et al, 2001; Hyun et al, 2017). Therefore, we assessed the colocalization of HP1 $\alpha$ with DAPI-rich regions in WT and Ago1_KO mESCs by IF (Fig 1C). Similarly, we observed a significant redistribution of $\mathrm{HP} 1 \alpha$ in Ago1_KO mESCs, away from the pericentromeric regions (Fig 1C). In addition, we noted a slight increase in HP1 $\alpha$ protein expression in Ag01_KO mESCs compared with WT cells (Fig S1F).

In conclusion, we observed a redistribution of both the repressive histone mark $\mathrm{H} 3 \mathrm{~K} 9 \mathrm{me} 3$ and the heterochromatin protein HP1 $\alpha$, away from pericentromeric regions in Ago1_KO mESCS.

\section{AGO1 complementation rescues the distribution of $\mathrm{H} 3 \mathrm{~K} 9 \mathrm{me} 3$ and $\mathrm{HP} 1 \alpha$ at pericentromeric regions}

To determine, whether the redistribution of H3K9me3 away from pericentromeric regions is specific to the loss of AGO1, we aimed to complement our Ag01_KO mESCs. We transfected the Ago1_KO2 mESC line with a vector expressing N-terminally HA-tagged AGO1. This vector additionally contains two selection markers, a GFP and a puromycin resistance gene. Cells expressing AGO1 were selected for a week for puromycin resistance, followed by FACS sorting for GFP (Fig S2A). Finally, we verified HA-AGO1 expression in the GFP sorted 
A

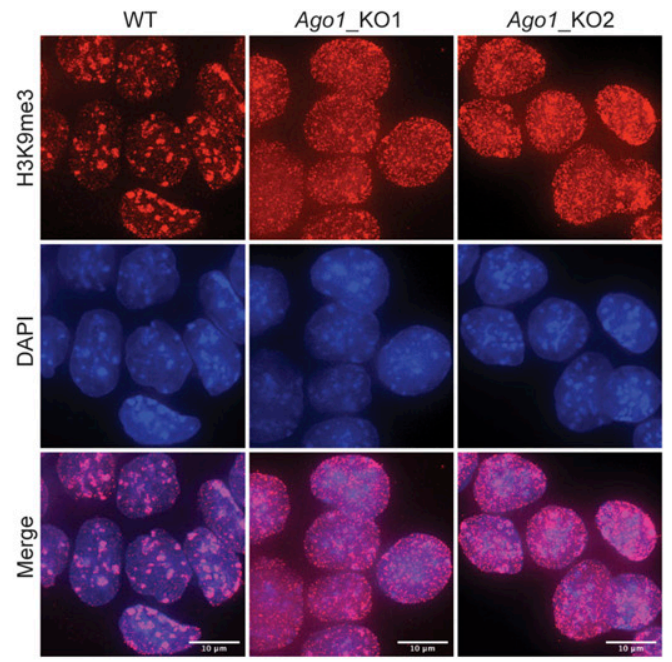

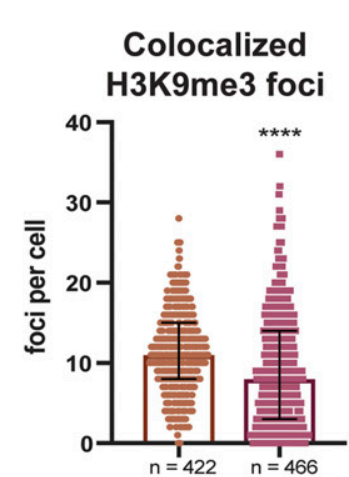

WT Ago1_KO
B

MajSat 1

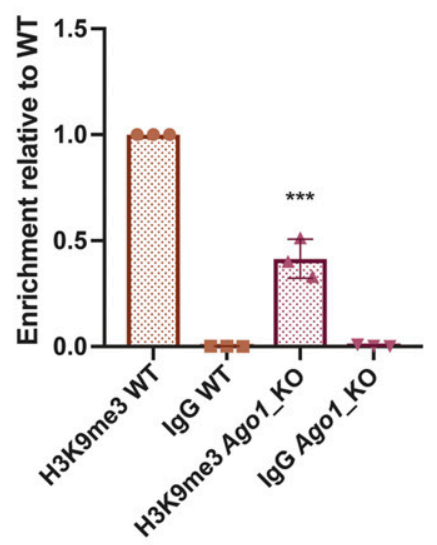

C
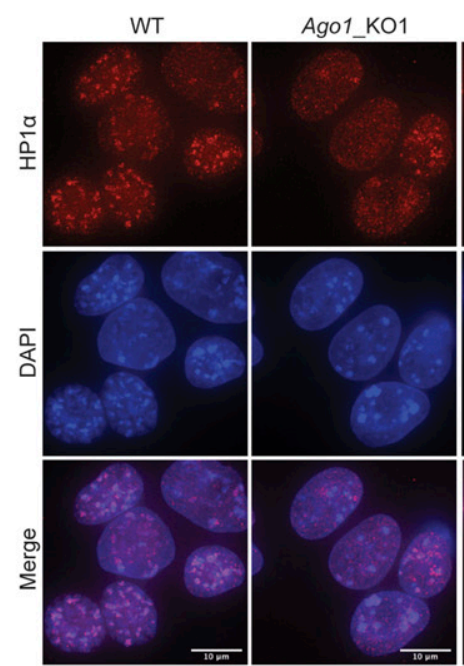

MajSat 2

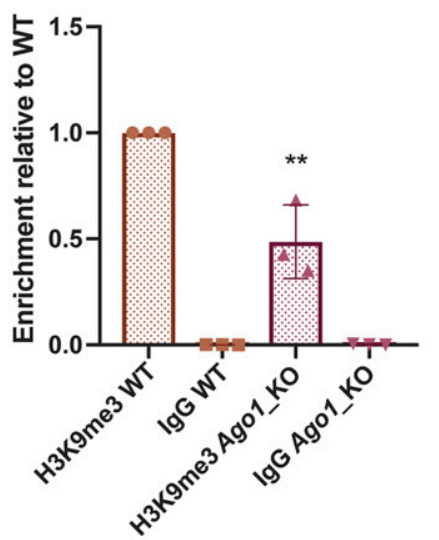

Figure 1. Distribution of H3K9me3 and HP1 $\alpha$ at pericentromeric regions in WT versus Ag01_KO mouse embryonic stem cells (mESCs).

(A) Left: representative IF images of H3K9me3 in WT and Ago1_KO $\mathrm{mESCs}$. Scale bar $=10 \mu \mathrm{m}$. Right: quantification of foci counts for H3K9me3 that colocalizes with DAPI regions in WT and Ag01_KO mESCs. Because of the bimodal distribution of H3K9me3 foci in Ag01_KO mESCS, the graph shows the median distribution with the interquartile range. ${ }^{* * * *}=P$-value $<0.0001$, Mann-Whitney test for $\mathrm{n}=3$ independent experiments. (B) ChIPqPCR in WT and Ag01_KO mESCs. Pull-downs were performed with an antibody against H3K9me3 and a control IgG antibody. qPCR has been performed on major satellite primer set 1 and 2 (Table S1). The enrichment was calculated over input and represented relative to the WT H3K9me3 pull-down. ${ }^{* * *}=$ $P$-value $<0.001$ and ${ }^{* *}=P$-value $<0.01$, unpaired $t$ test for $\mathrm{n}=3$ independent experiments. The Ag01_KO1 and Ag01_KO2 have been combined in this experiment. IgG error bars clipped at axis limit. (C) Left: representative IF images of HP1 in WT and Ago1_KO mESCs. Scale bar $=10 \mu \mathrm{m}$. Right: quantification of foci count for HP1 $\alpha$ that colocalizes with DAPI regions in WT and Ag01_KO mESCs. The graph shows the median distribution with the interquartile range. ${ }^{* *}=P$-value $<0.01$, Mann-Whitney test for $\mathrm{n}=3$ independent experiments. For the quantification Ag01_KO1, and Ag01_KO2 were combined. polyclonal cell population (mixed cell population) by IF and WB, and observed a partial rescue of AGO1 levels in the complemented cells (Figs 2A and S2B). We next tested whether the reintroduction of AGO1 could rescue the distribution of H3K9me3 foci at DAPI-rich regions and performed a co-staining for H3K9me3 and $\mathrm{HA}$ in WT, Ag01_KO and the Ag01_KO + HA-AGO1 cells (Fig 2A). The co-staining with $\mathrm{HA}$ allowed us to select cells re-expressing AGO1 at a proper level in the polyclonal population to perform the quantification. 
A

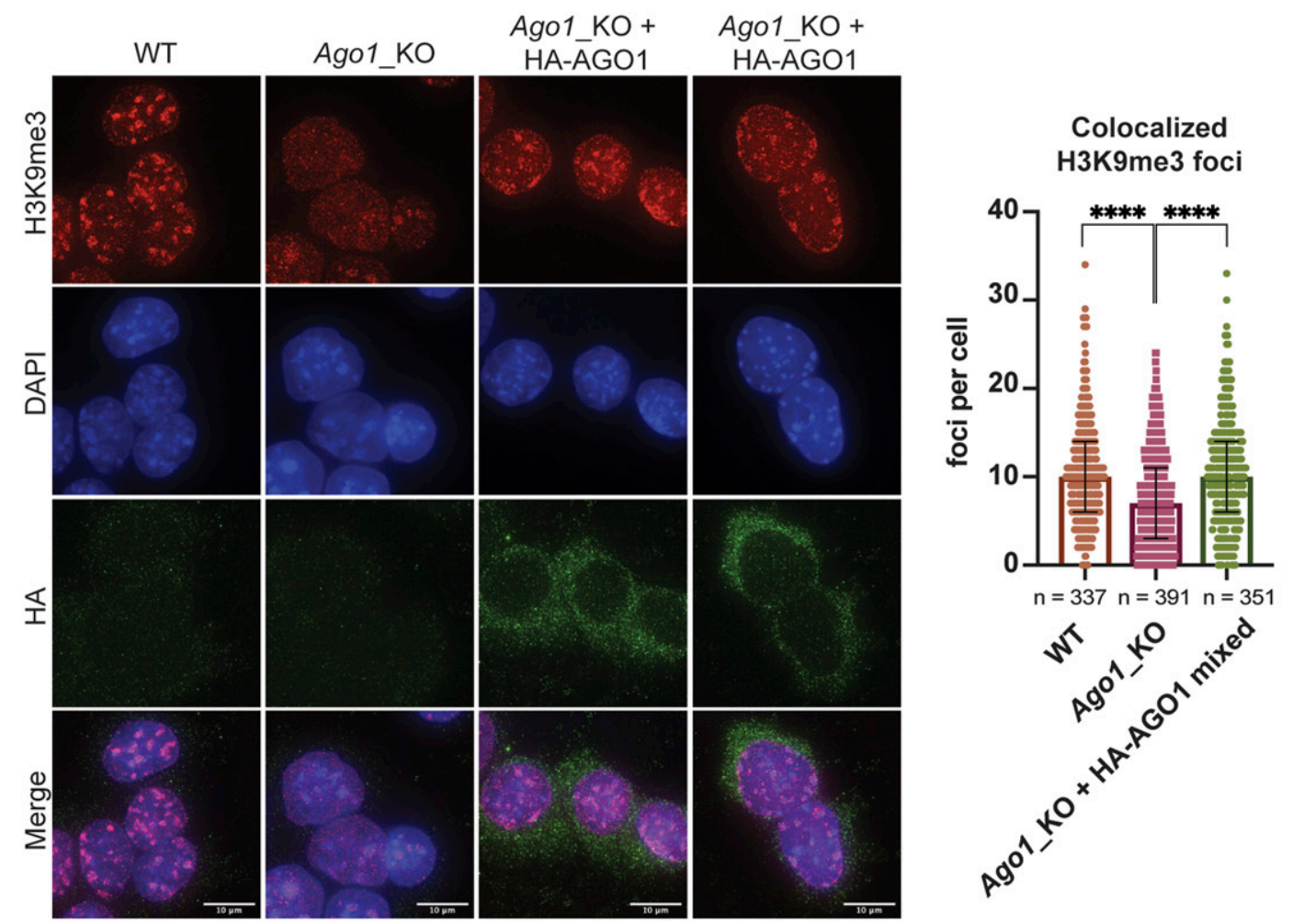

$\mathrm{B}$
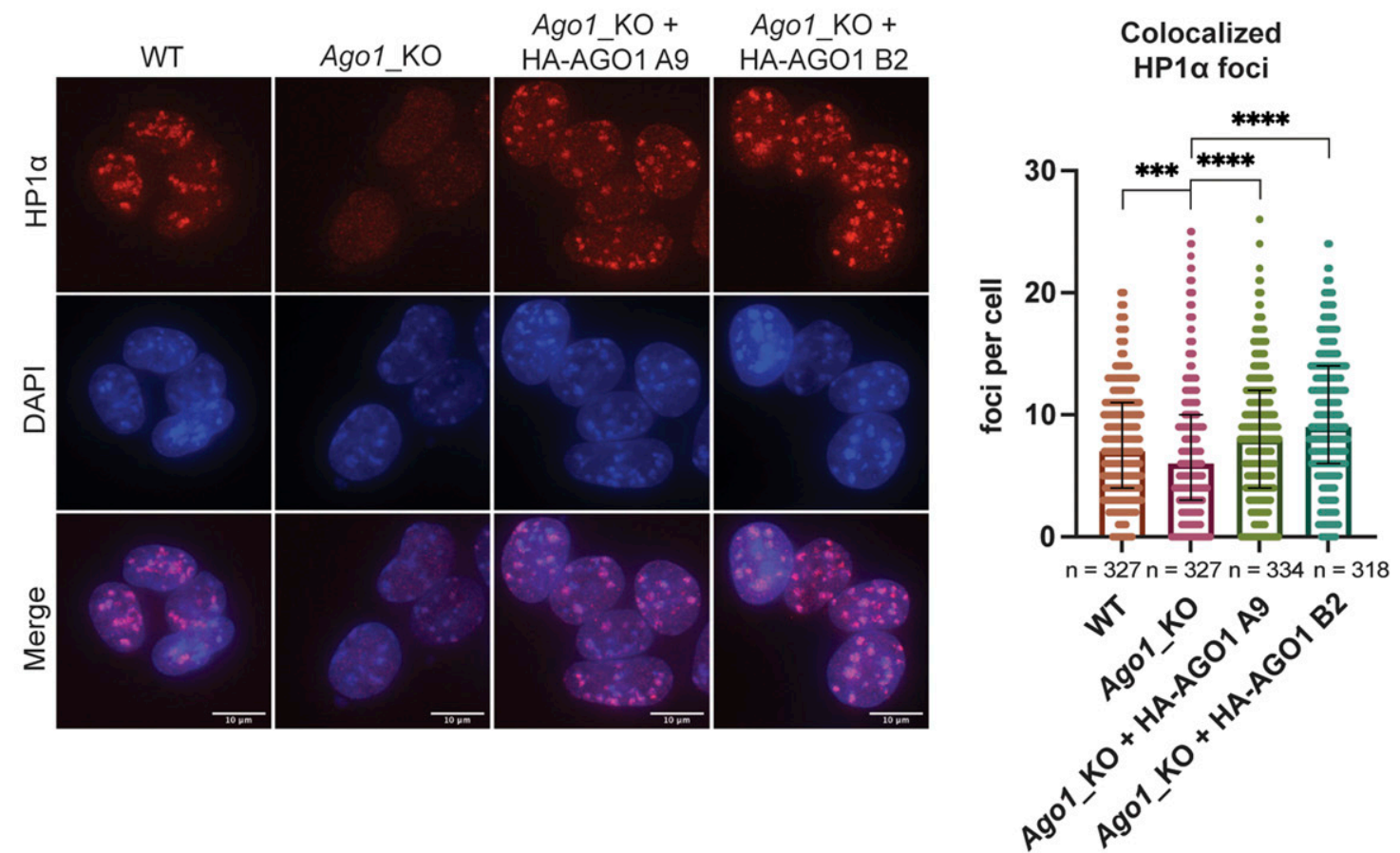

Figure 2. AGO1 complementation rescues the distribution of H3K9me3 and HP1 $\alpha$ at pericentromeric regions in Ago1_KO Mouse Embryonic Stem Cells (mESCs). (A) Left: representative IF images of H3K9me3 in WT, Ago1_KO and two representative images of Ago1_KO + 3x HA-AGO1 mixed population mESCs. Scale bar = 10 $\mu$ m. Right: quantification of foci count for H3K9me3 that colocalizes with DAPI regions in WT, Ago1_KO, and Ago1_KO + 3x HA-AGO1 mixed population mESCs. The graph shows the median distribution with the interquartile range. ${ }^{* \star *}=P$-value $<0.0001$, Mann-Whitney test for $n=3$ independent experiments. (B) Left: representative IF images of HP1 $\alpha$ in WT, Ag01_KO, and Ag01_KO + 3x HA-AG01 single clones A9 and B2. Right: quantification of foci count for HP1 $\alpha$ that colocalizes with DAPI regions in WT, Ago1_KO, and Ago1_KO + 3x HA-AGO1 single clones A9 and B2. The graph shows the median distribution with the interquartile range. ${ }^{\star \star \star *}=P$-value $<0.0001,{ }^{\star \star \star}=P$-value $<0.001$, Mann-Whitney test for $n=3$ independent experiments. 
We observed a significant rescue of the H3K9me3 distribution at the pericentromeric regions upon reintroduction of AGO1 in Ag01_KO mESC line (Fig 2A).

Because of the nuclear pre-extraction step necessary for proper visualization of $\mathrm{HP} 1 \alpha$ by IF, we were not able to simultaneously assess the distribution of HP1 $\alpha$ along with HA-AGO1 in the complemented polyclonal population. To solve this issue, we selected monoclonal clones using GFP as a marker by FACS sorting (Fig S2C). Two clones (B2 and A9) were kept for further analysis as they expressed HA-AGO1 at similar (B2) or at a higher level (A9) than the complemented polyclonal population of cells previously analyzed (Fig S2D). We then assessed the distribution of HP1 $\alpha$ in WT, Ago1_KO and Ag01_KO complemented clones by IF and, observed a similar significant rescue of the HP1 $\alpha$ distribution at the pericentromeres upon reintroduction of AGO1 in Ag01_KO mESC single clones (Fig 2B).

In conclusion, the reintroduction of AGO1 in Ago1_KO mESCS partially rescue the mislocalization of both H3K9me3 and HP1 $\alpha$ at pericentromeres.

\section{Major satellite transcripts are up-regulated in Ago1_KO mESCs}

In mouse, pericentromeric heterochromatin regions are characterized by AT-rich tandem repeats, known as major satellite repeat sequences. Major satellites consist of $234 \mathrm{bp}$ tandem repeat sequences that can stretch over several kilobases (Guenatri et al, 2004; Komissarov et al, 2011). The minor satellite sequences adjacent to the major satellites are localized to the centromeric part of the chromosome (Fig S3A) (Vissel \& Choo, 1989; Guenatri et al, 2004). Even though pericentromeric regions are marked by repressive heterochromatin marks, pericentromeric transcripts, such as major satellite transcripts, have been previously reported to be expressed in vivo during mouse early development and also in vitro in mESCs (Fig S3A) (Rudert et al, 1995; Lehnertz et al, 2003; Probst et al, 2010).

To assess whether the depletion of Ago1 affects major satellite transcripts, we performed an RNA FISH (Fig 3A). We observed a stronger signal and significantly more foci corresponding to major satellites in Ag01_KO compared with WT mESCs (Figs 3A and S3A). To confirm that the increase in foci number per cell corresponding to transcripts derived from major satellites is specific to the loss of AGO1 in mESCS, we performed RNA FISH in the previously derived single complemented clones A9 and B2. We observed that the reintroduction of AGO1 in Ago1_KO mESCs could also partially rescue the RNA FISH signal in the single complemented clones (Fig $3 A$ ). To better quantify the amount of major satellite transcripts in all cell lines, we then measured their relative expression using a stringent RT-qPCR protocol in WT, Ago1_KO, and Ago1_KO complemented clones (see the Materials and Methods section and Fig S3B). We detected a significant up-regulation of major satellite mRNAs in the Ago1_KO mESCs using two independent primer pairs and this up-regulation was decreased by half upon reintroduction of AGO1 in Ago1_KO mESC clones (Fig 3B).

Finally, to better understand whether the up-regulation of major satellite transcripts was linked to changes at the chromatin level, we analyzed the IF images for the H3K9me3 staining and quantified the number of DAPI foci (chromocenters) in WT versus Ago1_KO
mESCs. We observed no decrease in DAPI foci formation in Ago1_KO compared with WT mESCs (Fig S3C). In addition, we also performed DNA FISH for the major satellite repeats in these two cell lines. The major satellite DNA FISH signal was similar between WT and Ag01_KO mESCS and we did not detect a more dispersed signal for the major satellites in Ago1_KO mESCs, indicating that the overall structures of chromocenters is preserved in Ago1_KO mESCS (Fig S3D).

In summary, we observed that upon Ago1 depletion, major satellite transcripts are up-regulated in MESCs and that their expression can be partially rescued by the re-expression of AGO1. Furthermore, this phenotype was not accompanied by the loss of chromocenters structure, as they could still form normally in Ag01_KO mESCS.

\section{MiRNAs are involved in the regulation of major satellites in mESCs}

We then attempted to identify the molecular mechanism causing the up-regulation of major satellite transcripts in Ago1_KO mESCS. The AGO proteins are best known for their role in post-transcriptional silencing via miRNAs (Meister, 2013). To investigate the role of miRNAs in constitutive heterochromatin distribution in $\mathrm{mESCS}$, we performed IF of H3K9me3 and HP1 $\alpha$ in Drosha_KO mESCS (Cirera-Salinas et al, 2017), generated in the same background than our Ago1_KO mESCS. Interestingly, we observed a strong redistribution of H3K9me3 and HP1 $\alpha$ away from the pericentromeric regions in Drosha_KO compared with WT mESCS, suggesting a role for miRNAs in the regulation of pericentromeric regions in mESCs (Fig 4A and B). In addition, we observed an up-regulation of HP1 $\alpha$ in Drosha_KO mESCs (Fig S4A), suggesting that HP1 $\alpha$ itself might be directly regulated by miRNAs.

MiRNA gene regulation is usually taking place in the cytoplasm of the cells (Bartel, 2018). Nevertheless, AGO2 was previously shown to shuttle into the nucleus (Nishi et al, 2013) and be enriched in mESC nucleus (Sarshad et al, 2018). Accordingly, we sought to assess the subcellular distribution of AGO1 in mESCs. Using well-established biochemical assays (Gagnon et al, 2014b), we performed cytoplasmic/ nucleoplasmic/chromatin fractionation of WT mESCs and analyzed the abundance of AGO1 in these three fractions by WB (Fig 4C). We observed that the most of the AGO1 localized to the cytoplasm, whereas only around $10-15 \%$ of AGO1 is present in the nuclear fraction and even less than $3 \%$ is found in the chromatin fraction (Fig 4C). Cross-contamination was controlled for by using specific subcellular markers to validate the purity of the different fractions (Fig 4C). These results led us to hypothesize that, like AGO2, AGO1 loaded with miRNA is able to shuttle in the nucleus of mESCs and might target specific transcripts in the nucleus. We therefore attempted to identify whether miRNAs target major satellite transcripts in mESCs. Most major satellite annotations, as obtained from RepeatMasker and Dfam (Smit et al, 2013; Bao et al, 2015; Storer et al, 2021), were not properly mapped to any of the chromosomes and therefore annotated in unmapped genomic contigs (Fig S4B). In accordance with reports of major satellite sequences being several kilobases long, we selected those annotations that mapped to regions of at least 20 kbps of length, and focused on major satellite regions that mapped to chromosome X, 9 and the contigs JH583204.1 and GL456383.1. We searched for miRNAs with high confidence seed matches within these sequences and identified three miRNAs from the miR-30 family 
A

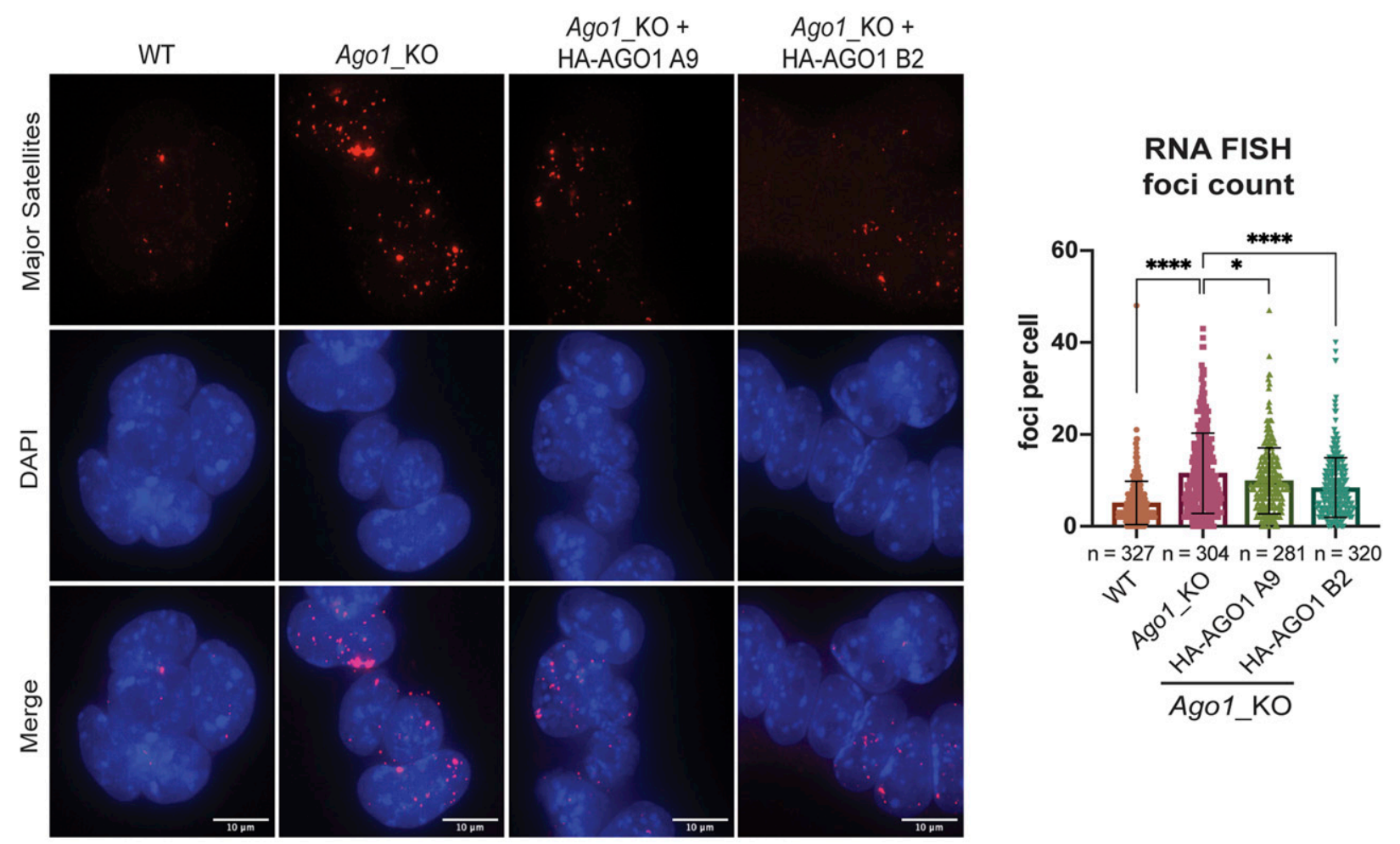

B

MajSat 1

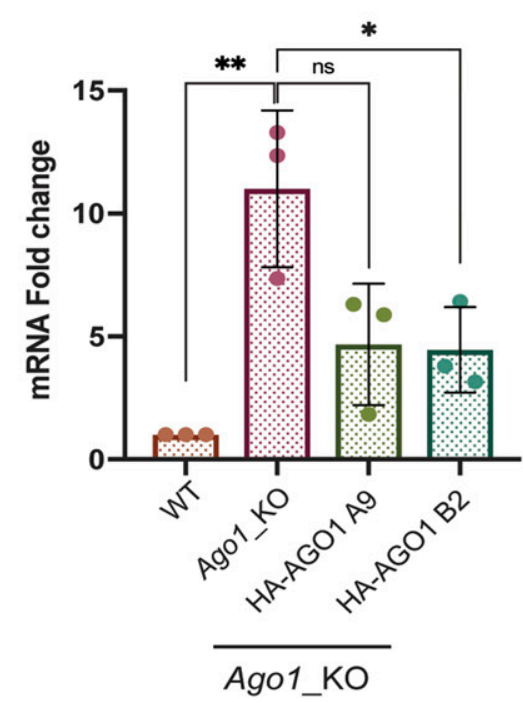

MajSat 2

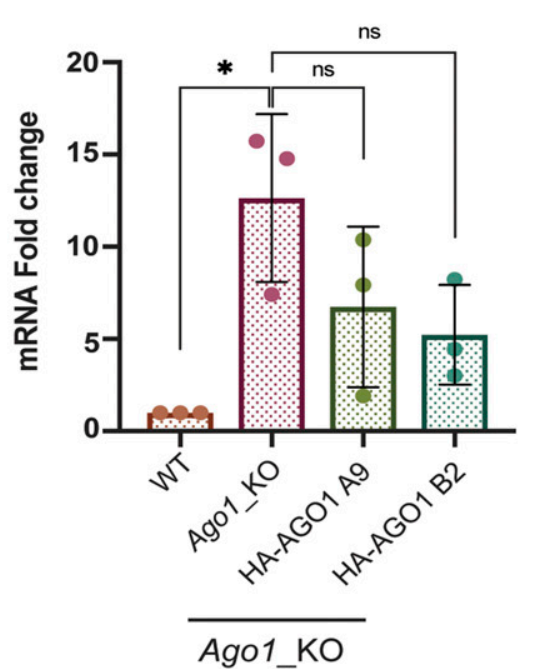

Figure 3. Major satellite transcripts are up-regulated in Ag01_KO mouse embryonic stem Cells (mESCs).

(A) Left: representative images of major satellite RNA FISH in WT, Ag01_KO, and two HA-AGO1 single clones, A9 and B2. Scale bar = $10 \mu$ m. Right: quantification of major satellite RNA FISH foci count in WT and Ago1_KO mESCs two HA-AGO1 single clones, A9 and B2. The graph shows the mean distribution with standard deviations. ${ }^{* * * *}=$ P-value $<$ 0.0001 and ${ }^{* * * *}=P$-value $<0.05$ unpaired $t$ test for $n=3$ independent experiments. (B) RT-qPCR results for major satellite primer set 1 and 2 (Table S1) in WT and Ago1_KO mESCS and two HA-AG01 single clones, A9 and B2. ${ }^{*}=P$-value $<0.05$ and ${ }^{* *}=P$-value $<0.01$, ns, not significant, unpaired $t$ test for $n=3$ independent experiments.

having a high number (more than 500) of 8mer binding sites (BS) within major satellite sequences (Fig 4D). In addition, two other miRNAs miR-139-5p and mR-6989-3p showed significant predicted BS. However, both these had much fewer binding sites compared with the miR-30-3p family (Fig 4D). Whereas, miR-139-5p has around
200 BS for the annotated region on the $X$ chromosome, it has basically no BS for the other three regions. Also, miR-6989-3p has only around $100 \mathrm{BS}$ in two contigs and even less in the others (Fig 4D).

The miR-30 family is composed of six pre-miRNAs (miR-30a, miR30b, miR-30c-1, miR-30c-2, miR-30d, and miR-30e) located on three 
A

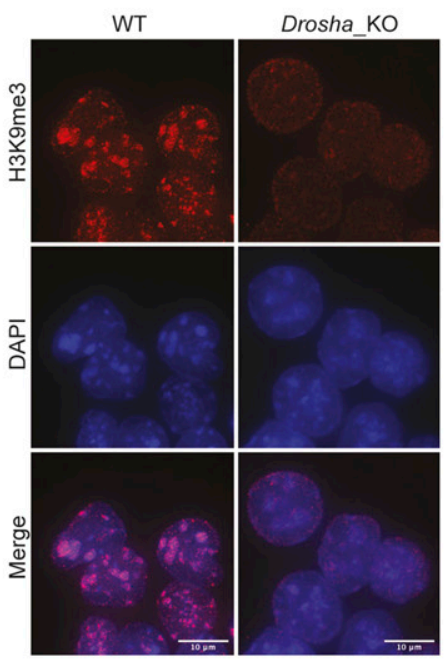

B

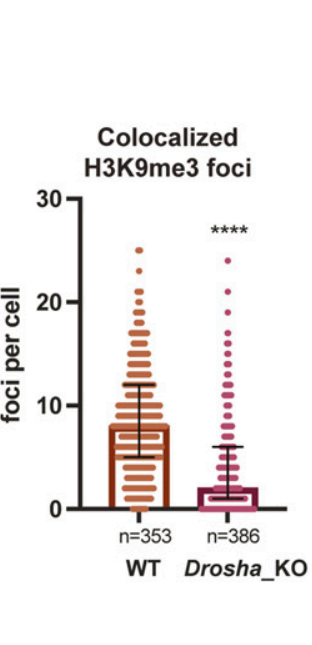

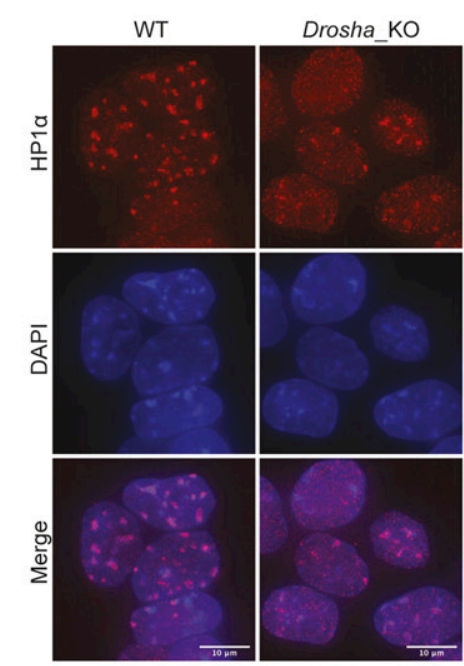

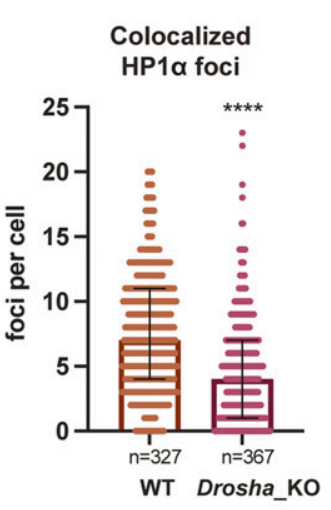

C

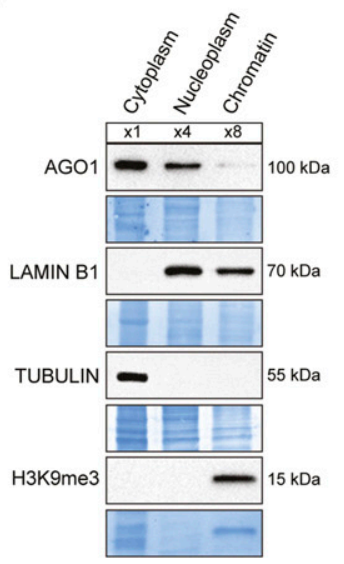

E

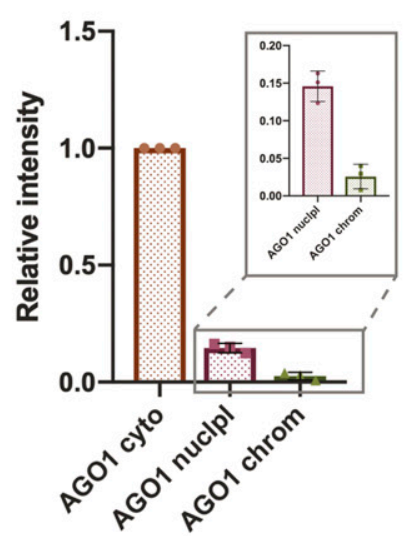

$\mathrm{F}$

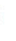

Top 5 miRNA with respect to

"Number of 8 mer binding sites in the satellite regions"

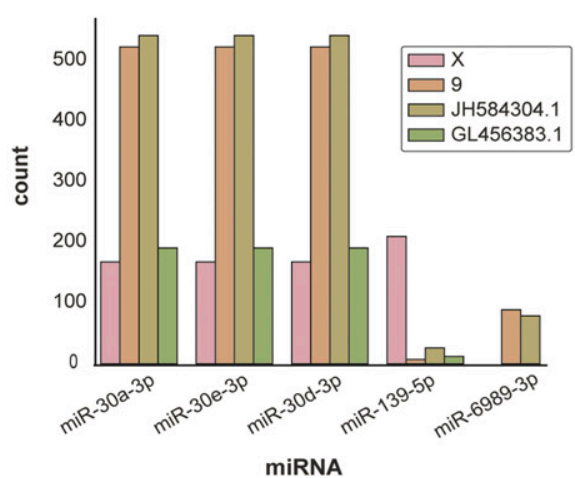

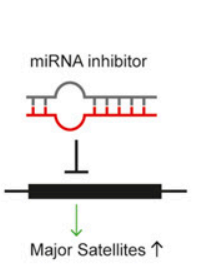
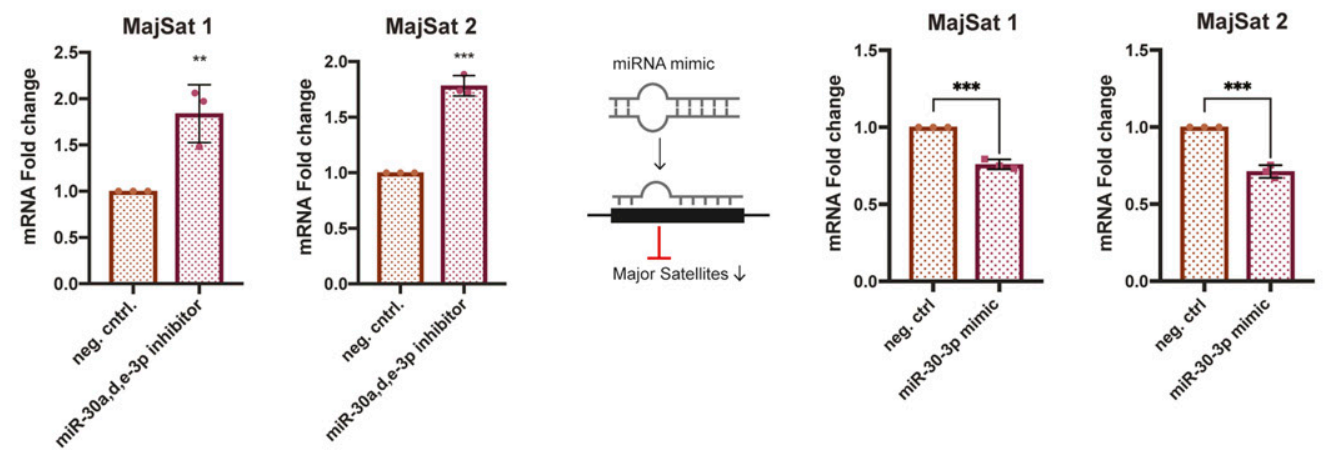

Figure 4. miRNAs are involved in the regulation of major satellite transcripts.

(A) Left: representative IF images of H3K9me3 in WT and Drosha_KO mouse embryonic stem cells (mESCs). Scale bar $=10 \mu \mathrm{m}$. Right: quantification of foci count for H3K9me3 that colocalizes with DAPI regions in WT and Drosha_KO mESCs. The graph shows the median distribution with the interquartile range. $* * * * P$-value $<0.0001$, Mann-Whitney test for $n=3$ independent experiments. (B) Left: representative IF images of HP1 $\alpha$ in WT and Drosha_KO mESCs. Right: quantification of foci count for HP1 $\alpha$ that colocalizes with DAPI regions in WT and Drosha_KO mESCS. The graph shows the median distribution with the interquartile range. ${ }^{* * *}=P$-value $<0.0001$, Mann-Whitney test for $n=3$ independent experiments. (C) Representative Western blots for the fractionation of WT mESCs to visualize AGO1 subcellular localization and 
different chromosomes, which are all expressed in WT mESCs (Fig S4C and D and Table S2). Whereas all the mature miR-30-5p share the same seed sequence, only miR-30a-3p, miR-30d-3p, and miR$30 e-3 p$ have identical seeds, which match the major satellite sequences (Figs 4D and S4E). In addition, by re-analyzing our published AGO1 RNA immunoprecipitation and sequencing (RIP-seq) data (Ngondo et al, 2018), we identified that these three miRNAs are preferentially loaded into AGO1 (Fig S4F and Table S2). To investigate a possible regulation of major satellite transcripts by miR30a-3p, miR-30d-3p, and miR-30e-3p, we used miRNA inhibitors against the three miRNAs in WT mESCs. We transfected WT mESCS either with a negative control inhibitor or with a pool of miR-30a-3p, miR-30d-3p, and miR-30e-3p inhibitors. We monitored the major satellite transcripts level $36 \mathrm{~h}$ after transfection by RT-qPCR and identified an increase in around twofold upon transfection with the miR-30a-3p, miR-30d-3p, and miR-30e-3p inhibitors compared with the negative control (Fig 4E). Similarly, transfecting WT mESCs with a miR-30-3p mimic significantly decreased major satellite transcript levels (Fig 4F). Taken together, these results indicate a role for the miR-30a-3p, miR-30d-3p, and miR-30e-3p in fine-tuning major satellite transcript levels.

\section{Discussion}

Since the discovery that AGO proteins can localize to the nucleus in mammalian cells, numerous studies have attempted to describe their nuclear functions (Meister, 2013). Although in human cells, nuclear AGO's have been linked to functions in transcriptional gene regulation, splicing, chromatin organization, and double-strand break repair (Janowski et al, 2006; Kim et al, 2006; Li et al, 2006; Ameyar-Zazoua et al, 2012; Hu et al, 2012; Huang et al, 2013; Alló et al, 2014; Cho et al, 2014; Gao et al, 2014; Agirre et al, 2015; Portnoy et al, 2016; Wang \& Goldstein, 2016; Shuaib et al, 2019), little is known about their role during early embryonic development.

In this study, we aimed to assess a possible role for AGO1 in the distribution of constitutive heterochromatin in mESCS. AGO1 had previously been reported to interact with RNA Polymerase II in human cells, where AGO1 was linked to chromatin and active promoters (Huang et al, 2013; Alló et al, 2014; Shuaib et al, 2019). We therefore decided to conduct a genetic approach by depleting Ago1 from WT MESCs (Fig S1A and B) and assessed by immunofluorescence the localization of the repressive histone mark H3K9me3 and the heterochromatin protein HP1 $\alpha$ in the mutant cell lines compared with WT mESCs (Fig 1). Surprisingly, we observed a redistribution of both H3K9me3 and HP1 $\alpha$ away from pericentromeric regions in Ago1_KO mESCS (Fig 1). The redistribution of H3K9me3 and $\mathrm{HP} 1 \alpha$ was found to be specific to the loss of AGO1, as reintroducing AGO1 could rescue the phenotype (Fig 2). We questioned whether major satellites residing within pericentromeric regions are up-regulated at the transcript level in Ag01_KO mESCs. Indeed, we observed an increase in pericentromeric major satellite transcripts in Ag01_KO mESCs by RT-qPCR and RNA FISH, which could again be rescued by reintroducing AGO1 into Ag01_KO mESCS (Fig 3). This increase was not caused by a change in the number of chromocenters, as was confirmed by DNA FISH (Fig S3C and D). Finally, we wondered whether AGO1 could regulate pericentromeric transcripts by a miRNA-mediated mechanism and observed similar delocalization of both H3K9me3 and HP1 $\alpha$ away from pericentromeric regions also in Drosha_KO mESCs, suggesting a role for miRNAs (Fig 4A and B). Using computational analysis, we identified that miR-30a-3p, miR-30d-3p, and miR-30e-3p might target major satellite transcripts and that manipulating the amount of these miRNAs in WT mESCs using inhibitors or mimics inversely regulates major satellite transcript levels to some extent (Fig 4D-F). In addition, by analyzing the subcellular distribution of the AGO1, we found that a small fraction (10-15\%) localized to the nucleus, leading us to the hypothesis that AGO1 loaded with miR-30-3p might directly regulate major satellite transcripts. Of note, the regulation observed here using mimics or inhibitors (Fig $4 \mathrm{E}$ and $\mathrm{F}$ ) was lower (twofold) than the one observed comparing WT and Ago1_KO mESCS (10-fold) (Fig 3B). We also observed an upregulation of HP1 $\alpha$ at protein level in Ag01_KO (Fig S1F) and Drosha_KO mESCs (Fig S4A), leading us to propose that HP1 $\alpha$ might also be directly regulated by miRNAs in mESCs (as also suggested by several miRNA binding sites in its 3'UTR and AGO2-binding sites [Schäfer et al, 2021 Preprint]). It would be interesting to further investigate this direct regulation of HP1 $\alpha$ by miRNAs in a follow-up study.

An involvement of AGO proteins loaded with small RNAs in the regulation of pericentromeric regions has previously been reported in Schizosaccharomyces pombe, where Ago1 loaded with siRNAs is guided to pericentromeres (Verdel et al, 2004). Ago1 together with Tas3 and Chp1 forms the RNA-induced transcriptional silencing complex (RITS). The RITS is guided to centromeric repeats by siRNAS, which are derived from this region. Targeting the RITS complex to centromeric repeats is needed for the localization of the HP1a homolog Swi6 and the nucleation of heterochromatin H3K9me at these sites (Motamedi et al, 2004; Verdel et al, 2004; Bühler et al, 2006; Goto \& Nakayama, 2012). However, our findings differ from the ones in yeast as we did not identify any small RNAs, derived from pericentromeric regions to be loaded in AG01. However, there have been reports suggesting the presence of small RNAs from pericentromeric regions in mammalian cells (Kanellopoulou et al, 2005; Hsieh et al, 2011), we found that AG01 in mESCs is probably guided to major satellite transcripts by specific miRNAs, miR-30a-3p, miR30d-3p, and miR-30e-3p.

Importantly, even though we identified a decrease in H3K9me3 at pericentromeric regions upon the depletion of Ago1 in $\mathrm{mESCS}$, this was accompanied by only a small impact on the global

quantification of $\mathrm{n}=3$ independent experiments. LAMIN B1 (nucleoplasm and chromatin), TUBULIN (cytoplasm), and H3K9me3 (chromatin) were used as subcellular markers. (D) Representation of the top 5 miRNAs with 8mer binding sites targeting major satellite sequences located on chromosomes X, 9 and the genomic contigs JH584304.1 and GL456383.1. (E) RT-qPCR results for major satellite primer sets 1 and 2 (Table S1) in WT mESCs transfected with a negative control inhibitor and a pool of inhibitors against miR-30a, d, e-3p. ${ }^{* *}=P$-value $<0.01$ and ${ }^{* * *}=P$-value $<0.001$, unpaired $t$ test for $n=3$ independent experiments. (F) RT-qPCR results for major satellite primer sets 1 and 2 (Table S1) in WT mESCs transfected with a negative control mimic and a miR-30-3p mimic. ${ }^{* * *}=P$-value $<0.001$, unpaired $t$ test for $n=3$ independent experiments. 
transcriptome and none regarding viability of Ag01_KO mESCS (Ngondo et al, 2018; Mueller et al, 2021 Preprint). Ngondo et al (2018), have reported that the depletion of Ago1 does not affect the cell cycle nor their potential to differentiate (Van Stry et al, 2012; Ngondo et al, 2018). It appears that the loss of HP1 $\alpha$ and H3K9me3 disturbs the environment more locally without affecting overall cell viability. Although we do not currently know how mESCs cope with this loss, it is possible that the plasticity of stem cells or the reestablishment of heterochromatin at pericentromeric regions upon differentiation may be required for survival. As several studies in human and cancer cells have already described a nuclear role for the AGO proteins, especially also for AGO1 (Janowski et al, 2006; Kim et al, 2006; Li et al, 2006; Ameyar-Zazoua et al, 2012; Hu et al, 2012; Huang et al, 2013; Alló et al, 2014; Cho et al, 2014; Agirre et al, 2015; Portnoy et al, 2016; Shuaib et al, 2019), it will be interesting to study whether the decrease of H3K9me3 at pericentromeric regions also occurs in these cell types. Interestingly, the up-regulation of major satellite transcripts in several cancer lines has already been described (Hall et al, 2012); however, we do not know whether this might be linked to a nuclear AGO1 function.

There are still open questions and further experiments required to identify the complete underlying molecular mechanism. How AGO1 regulates heterochromatin at pericentromeric regions and major satellite transcripts remains an open question. Our attempts to localize AGO1 at pericentromeric regions using IF or ChIP-qPCR approaches, using specific antibodies or AGO1-tagged cell lines, remained unsuccessful (data not shown). These negative results might come from the low amount of AGO1 in the nucleus (Fig 4C). New approaches or technical development are therefore required to precisely address AGO1 localization in the nucleus or chromatin in mESCs. Furthermore, to dissect a direct role of AGO1, it might be helpful in the future to assess whether AGO1 RNA binding activity is required for direct major satellite targeting via miRNAs.

In conclusion, our study reports a novel role for AGO1 in the nucleus of mESCs and we believe that these observations might help to motivate future research on the AGO proteins in early embryonic development.

\section{Materials and Methods}

\section{Mouse ESC lines}

WT E14 (129/Ola background), Ag01_KO, AGO1 complemented Ago1_KO, and Drosha_KO (Cirera-Salinas et al, 2017) mESCs were cultured in DMEM (Sigma-Aldrich), supplemented with 15\% FBS (Life Technologies), $100 \mathrm{U} / \mathrm{ml}$ LIF (Millipore), $0.1 \mathrm{mM}$ 2- $\beta$-mercaptoethanol (Life Technologies), and 1\% penicillin/streptomycin (SigmaAldrich). MESCs were cultured on $0.2 \%$ gelatin-coated culture flasks in the absence of feeder cells. The culture medium was changed daily and all cells were grown at $37^{\circ} \mathrm{C}$ in $8 \% \mathrm{CO}_{2}$.

\section{CRISPR/Cas9-mediated gene knockout}

The generation of the Ago1_KO1 cell line was previously published by (Ngondo et al, 2018). The Ag01_KO2 cell line was generated using a paired CRISPR/Cas9 approach, as described by Wettstein et al (2016). E14 mESCs were transfected with lipofectamine 2000 (Invitrogen) and the pX458-sgRNA_Ag01_5/6 (\#172470, \#172471; Addgene). After 48 h, GFP-positive cells were single sorted into 96-well plates (TPP). To confirm the deletion, genotyping at DNA level was performed, with the primers PS_Ago1_FW/RW_1 listed in Table S1. MESC clones were then amplified and the absence of AGO1 protein and RNA was additionally verified by Western blotting and RT-qPCR, respectively.

\section{Generation of Ag01_KO complemented cell lines}

For the rescue experiments, the AGO1 complemented Ag01_KO2 cells were obtained by stably transfecting the pMSCV_PIG_3xHAAG01 plasmid (\#170916; Addgene) with lipofectamine 3000 (Invitrogen). Cells were grown for 1 wk under puromycin selection and then sorted by FACS to select only GFP expressing cells. We sorted two mixed population into separate dishes of around 10,000-20,000 cells. The mixed populations were expanded and the expression of HA-AGO1 was tested by Western blot and Immunofluorescence (Figs $2 A$ and S2A and B). For single clone generation, single cells expressing GFP were sorted into a 96-well plate and expanded. The expression of HA-AGO1 was tested by Western blot (Fig S2C and D).

\section{Cytoplasmic/nucleoplasmic/chromatin fractionation}

Cytoplasmic/nucleoplasmic/chromatin fractionation was performed after the protocol of Gagnon et al (2014b). Cells were grown to near confluency in two $75 \mathrm{~cm}^{2}$ (T75) flasks (TPP). 10 millions of WT mESCs were used. Freshly harvested cells were incubated for $10 \mathrm{~min}$ in ice-cold Hypotonic lysis buffer complemented with EDTA-free protease inhibitor cocktail (Roche) and Phosphatase inhibitor cocktail (Roche). After centrifugation ( $800 \mathrm{~g}$ for $8 \mathrm{~min}$ at $4^{\circ} \mathrm{C}$ ) the cytoplasmic fraction was transferred to a new tube containing $5 \mathrm{M} \mathrm{NaCl}$. Pellets were washed four times with Hypotonic lysis buffer (200g for $2 \mathrm{~min})$. After the last wash ice-cold modified Wuarin-Schipler buffer (MWS) $(10 \mathrm{mM}$ Tris- $\mathrm{HCl}$, [pH 7.0], $4 \mathrm{mM}$ EDTA, $0.3 \mathrm{M} \mathrm{NaCl}, 1 \mathrm{M}$ urea, and 1\% [vol/vol] IGEPAL-C630), complemented with EDTA-free protease inhibitor cocktail and Phosphatase inhibitor cocktail, was added and after vortexing, incubated for $15 \mathrm{~min}$ on ice. After centrifugation $\left(1,000 \mathrm{~g}\right.$ for $5 \mathrm{~min}$ at $\left.4^{\circ} \mathrm{C}\right)$, the nucleoplasmic fraction was transferred to a new tube. The chromatin pellet was washed twice with MWS buffer, vortexed, incubated on ice for $5 \mathrm{~min}$, and centrifuged at $500 \mathrm{~g}$ for $3 \mathrm{~min}$ at $4^{\circ} \mathrm{C}$. Ice-cold NLB was added to the chromatin pellet, which was sonicated twice at $20 \%$ for $15 \mathrm{~s}$ with 2 min incubations on ice in between. The three fractions were centrifuged for $15 \mathrm{~min}$ at 18,000 $\mathrm{g}$ and the supernatant was transferred to a new tube.

The fractions were then analyzed by Western blot. To ensure proper representation of all the fractions, more of the nuclear $(\times 4)$ and the chromatin $(\times 8)$ fraction were loaded (Fig 4C).

Analysis of the Western blot signal was performed using ImageLab (Bio-Rad Laboratories). The intensity of the bands was calculated relative to the WT band. The intensities of the nuclear and chromatin fractions were adjusted according to 
the additional loading and the fact that they were resuspended in half the amount of buffer compared with the cytoplasm.

\section{Western blot analysis}

Total cellular proteins were extracted using RIPA lysing buffer (50 mM Tris-HCL, pH 8.0, $150 \mathrm{mM} \mathrm{NaCl,} \mathrm{1 \%} \mathrm{IGEPAL-CA630,} \mathrm{0.5 \%}$ sodium deoxycholate, and $0.5 \%$ sodium dodecyl sulfate supplemented with EDTA-free protease inhibitor cocktail [Roche]). Protein concentration was determined by a Bradford assay (BioRad Laboratories). Proteins were separated on an SDS-PAGE gel and transferred to a polyvinylidene difluoride membrane (Sigma-Aldrich). Membranes were blocked for at least $30 \mathrm{~min}$ in blocking solution (5\% milk in 1X TBS-T: TBS, pH 7.6: 50 mM Tris-HCL, $150 \mathrm{mM} \mathrm{NaCl}$, and $0.1 \%$ Tween 20) and incubated overnight with primary antibodies diluted in blocking solution at $4^{\circ} \mathrm{C}$. Primary antibodies used were: HP1 $\alpha$ (\#2616, 1:2,000; CST), AGO1 (\#5053, 1: 2,000; CST), LAMIN B1 (ab16048, 1:10,000; Abcam), TUBULIN (T6199, 1: 10,000; Sigma-Aldrich), H3K9me3 (ab8898, 1:2,000; Abcam), and HA (3F10, 1:2,000; Roche).

After washing three times in $1 \times$ TBS-T for $10 \mathrm{~min}$, membranes were incubated with the secondary antibody for $1 \mathrm{~h}$ at room temperature (rabbit-IgG HRP-linked 1:10,000; Cell Signaling Technology [\#7074], mouse-IgG HRP-linked 1:10,000; Cell Signaling Technology [\#7076], rat-IgG HRP-linked 1:10,000 [\#7077]). After incubation, membranes were washed again three times 10 min in $1 X$ TBS-T and developed using the Clarify Western ECL substrate kit (Bio-Rad) or SuperSignal West Femto (Thermo Fisher Scientific). Membranes were imaged using the ChemiDoc MP imaging system (Bio-Rad Laboratories).

Analysis of the Western blot signal was performed using ImageLab (Bio-Rad Laboratories). Coomassie or TUBULIN was used as normalizer. Intensities of the bands were calculated relative to the WT band.

\section{Immunofluorescence and analysis}

Approximately 100,000 cells were plated the night before into sixwell plates (TPP), containing coverslips coated with fibronectin (1:100 in 1x PBS; Merck). The next day, cells were washed once with 1× PBS.

For the H3K9me3 and HA staining, cells were fixed with ice-cold Methanol for $10 \mathrm{~min}$ at $-20^{\circ} \mathrm{C}$. After fixation they were washed three times with 1× PBS and blocked for 20 min in blocking solution (1\% BSA in $1 X$ PBS-Tween 20 [0.1\%]).

For the HP1 $\alpha$ staining, a nuclear pre-extraction was performed. Cells were washed once with ice-cold $1 \times$ PBS for 3 min on ice and then incubated in CSK buffer (0.1\% Triton X-100, 10 mM PIPES, 100 $\mathrm{mM} \mathrm{NaCl}, 3 \mathrm{mM} \mathrm{MgCl}$, and $300 \mathrm{mM}$ Sucrose) for $3 \mathrm{~min}$, also on ice. Afterwards cells were washed once with $1 \times$ PBS and fixed with $3.7 \%$ formaldehyde (Sigma-Aldrich) for $10 \mathrm{~min}$ at room temperature. After fixation, cells were washed twice with $1 \times$ PBS for 5 min at room temperature and then permeabilized with CSK buffer (same as above) for 4 min on ice. After two additional wash steps with 1× PBS at room temperature, cells were blocked in blocking solution (1\% BSA in 1× PBS-Tween 20 [0.1\%]) for $20 \mathrm{~min}$ at room temperature.
After blocking, cells were incubated with the primary antibodies

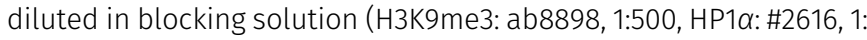
200; CST, HA: 3F10, 1:250; Roche) for $1 \mathrm{~h}$ at room temperature. Coverslips were washed three times for $5 \mathrm{~min}$ at room temperature with $1 \times$ PBS-Tween 20 (0.1\%). Then, cells were incubated with secondary antibodies diluted in blocking solution (1:2,000; Invitrogen) for $1 \mathrm{~h}$ at room temperature in the dark. Again, coverslips were washed three times for $5 \mathrm{~min}$ at room temperature with $1 \times$ PBS-Tween 20 (0.1\%) and once with $1 \times$ PBS. Counterstain with DAPI $(0.1 \mu \mathrm{l} / \mathrm{ml})$ in $1 \times$ PBS was performed for $4 \mathrm{~min}$ at room temperature. Cells were washed once with $1 \times$ PBS and mounted on microscopy slides on a drop of antifade medium (Vectashield; Vector Laboratories). Slides were imaged on a DeltaVision Multiplexed system with an Olympus IX71 inverse microscope equipped with a $60 \times 1.4 \mathrm{NA}$ DIC Oil PlanApoN objective and a pco.edge 5.5 camera, provided by the ScopeM facility of ETH.

For image analysis, deconvolved images were processed with Fiji (Schindelin et al, 2012). A Z-projection of the Max intensity has been performed for each image and used for further analysis. Foci count and intensity analysis was performed on the Z-projected images, with the help of CellProfiler (Mcquin et al, 2018). In CellProfiler, nuclei were identified by using the IdentifyPrimaryObjects module and the Otsu thresholding method. Nuclei were edited manually and the DAPI and H3K9me3 was enhanced with the EnhanceOrSupressFeatures module to detect speckles. Foci were identified by using IdentifyPrimaryObject. For the DAPI foci, the RobustBackground was used as a thresholding method and the threshold strategy was set to Global. Typical diameter of objects, in pixel units was set to 5-35. For the H3K9me3 foci the same thresholding method and strategy was used, but the typical diameter of objects, in pixel units was set to 7-35. Foci were related to the edited nuclei and the H3K9me3 foci were related to the DAPI foci. The MeasureObjectIntensity module was used to measure object intensity and foci count and results were exported to a csv file. Overlaid objects were saved as png.

\section{RNA extraction and quantitative RT-qPCR analysis}

RNA extraction and RT PCR analysis has been performed as previously described by Bodak and Ciaudo (2016). Briefly, total RNA from mESC pellets was extracted using Trizol (Life Technologies) according to standard protocols. RNA quality was checked, by running $1 \mu \mathrm{g}$ on a $1 \%$ agarose gel (Sigma-Aldrich).

For qPCR on major satellite transcripts, $20 \mu \mathrm{g}$ of RNA was treated twice with $1 \mathrm{U}$ of DNasel (QIAGEN) per $\mu$ g of RNA. RiboLock was added to reduce RNA degradation. DNase-treated RNA was purified using Direct-zol RNA mini prep kit (Zymo Research). Reverse transcription and qPCR were performed as described above. Primers are listed in the Table S1.

\section{RNA FISH}

The plasmid pCR4 Maj9-2 (a kind gift from the Almouzni laboratory, originally from Lehnertz et al [2003]) was used to generate the RNA FISH probe by nick translation (Abott). In brief, $2 \mu \mathrm{g}$ of plasmid, $3 \mu \mathrm{l}$ of nick translation enzyme, $2.5 \mu \mathrm{l} 0.2 \mathrm{mM}$ red-dUTP, $5 \mu \mathrm{l} 0.1 \mathrm{mM} \mathrm{dTTP}$, 
$10 \mu \mathrm{l} 0.1 \mathrm{mM}$ dNTP mix, and $5 \mu \mathrm{l} 10 \times$ nick translation buffer were incubated for $15 \mathrm{~h}$ at $15^{\circ} \mathrm{C}$. Nick translation efficiency was checked for by running $3 \mu \mathrm{l}$ probe on a $1 \%$ agarose gel (Sigma-Aldrich). The rest of the probe was cleaned-up with the Zymoclean Gel DNA Recovery Kit (Zymo Research). The probe was dried down to $5 \mu \mathrm{l}$ using a speed vac and then resuspended in hybridization solution (50 $\mu \mathrm{l}$ Deionized Formamide, $10 \mu \mathrm{l}$ 20X SSC, $2 \mu \mathrm{l} 100 \mathrm{mg} / \mathrm{ml} \mathrm{BSA}, 20 \mu \mathrm{l}$ $50 \%$ Dextran Sulfate, $3 \mu \mathrm{l}$ Salmon Sperm, $10 \mu \mathrm{l} \mathrm{RVC}$ ). Before use, the probe was diluted 1:2 in hybridization solution.

Approximately 150,000 cells were plated the night before into sixwell plates, containing coverslips coated with fibronectin (1:100 in $1 \mathrm{X}$ PBS; Merck). A nuclear pre-extraction was performed. Cells were washed once with ice-cold $1 \times$ PBS for 3 min on ice and then incubated in CSK buffer (0.1\% Triton X-100, 10 mM PIPES, $100 \mathrm{mM} \mathrm{NaCl}$, $3 \mathrm{mM} \mathrm{MgCl}$, and $300 \mathrm{mM}$ sucrose) for $3 \mathrm{~min}$, also on ice. Afterwards, the cells were washed once with $1 \times$ PBS and fixed with $3.7 \%$ formaldehyde (Sigma-Aldrich) for $10 \mathrm{~min}$ at room temperature. After fixation, cells were washed twice with $1 \times$ PBS for $5 \mathrm{~min}$ at room temperature and then permeabilized with CSK buffer (same as above) for 4 min on ice. After two additional wash steps with 1× PBS at room temperature, cells were blocked in blocking solution (1\% BSA in 1X PBS and RVC [1 mM]) for $30 \mathrm{~min}$ at room temperature. Then coverslips were washed once with $2 \times$ SSC and dehydrated with ethanol (70\% EtOH for $3 \mathrm{~min}, 90 \% \mathrm{EtOH}$ for $3 \mathrm{~min}$, and $100 \% \mathrm{EtOH}$ for $3 \mathrm{~min}$ ). The probe was denatured for 5 min at $76^{\circ} \mathrm{C} .10 \mu \mathrm{l}$ of the denatured probe (diluted $1: 2$ in hybridization buffer) was spotted on a baked slide. The coverslips were air-dried and placed on the spotted probe. The coverslips were sealed with rubber cement and then incubated overnight at $37^{\circ} \mathrm{C}$ in a humid chamber. The next day, coverslips were washed twice with $50 \%$ formamide $/ 2 \times \mathrm{SSC}$ for $5 \mathrm{~min}$ at $37^{\circ} \mathrm{C}$ and then once for 5 min with $2 \times$ SSC at room temperature. Counterstain with DAPI $(0.1 \mu \mathrm{l} / \mathrm{ml})$ in $2 \times$ SSC was performed for $4 \mathrm{~min}$ at room temperature. Coverslips were washed again once in 2x SSC and once 1× PBS and then mounted on microscopy slides on a drop of antifade medium (Vectashield; Vector Laboratories). Slides were image on a DeltaVision Multiplexed system provided by the ScopeM facility of ETH as above.

For image analysis, deconvolved images were processed with Fiji (Schindelin et al, 2012). A Z-projection of the Max intensity has been performed for each image and used for further analysis. RNA Foci were counted by eye. All Z-projected images of one replicate were opened using the Fiji software and were set to the same intensity.

\section{DNA FISH}

DNA FISH was performed exactly as described for the RNA FISH. The only differences, are that no RVC was used in the blocking solution and the samples were denatured by incubating the slides at $76^{\circ} \mathrm{C}$ for 5 min once the coverslips were placed on the spotted probe and sealed with rubber cement.

\section{ChIP and ChIP-qPCR analysis}

Four million cells were plated the night before into a gelatin-coated $60.1 \mathrm{~cm}^{2}$ (B10) dish (TPP). For each condition, two B10 dishes were prepared in parallel. Cells were cross-linked with $1 \%$ formaldehyde in DMEM for $10 \mathrm{~min}$ at room temperature. The reaction was quenched with glycine (125 mM; PanReac Applichem) for 5 min at room temperature. Cells were washed once with ice-cold 1× PBS and then swelling buffer ( $5 \mathrm{mM}$ Hepes, $\mathrm{pH} 8,85 \mathrm{mM} \mathrm{KCl}, 0.5 \%$ IGEPALCA630, and protease inhibitor cocktail [Roche]) was added to the cells. Cells were scraped and transferred to a $15 \mathrm{ml}$ falcon (Greiner), where they were incubated for $15 \mathrm{~min}$ on ice. Cells were centrifuged 5 min at 250g (Eppendorf Centrifuge 5810R; Rotor A-4-81 [for Falcon tubes]) at $4^{\circ} \mathrm{C}$, to pellet the nuclei. Afterwards, nuclei were washed again with swelling buffer followed by another centrifugation $(250 \mathrm{~g}$ at $4^{\circ} \mathrm{C}$ for $\left.5 \mathrm{~min}\right)$. The nuclei pellet was lysed in $400 \mu \mathrm{l}$ RIPA buffer $1 \%$ SDS (1× PBS, 1\% IGEPAL-C630, 0.5\% sodium deoxycholate, $1 \%$ sodium dodecyl sulfate, and protease inhibitor cocktail [Roche]) and incubated on ice for $10 \mathrm{~min}$. The lysates were sonicated on a Bioruptor (Diagenode) for $30 \mathrm{~min}, 30 \mathrm{~s}$ on and $30 \mathrm{~s}$ off cycles at $4^{\circ} \mathrm{C}$. Lysates were centrifuged at max speed for $15 \mathrm{~min}$ at $4^{\circ} \mathrm{C}$. The supernatant was retrieved into a new $2 \mathrm{ml}$ Eppendorf tube and diluted 10 times with RIPA buffer 0\% SDS ( $1 \times$ PBS, 1\% IGEPAL-C630, 0.5\% sodium deoxycholate, and protease inhibitor cocktail [Roche]) to obtain a concentration of $0.1 \%$ sodium dodecyl sulfate. $10 \%$ of chromatin was taken away for Input calculation, the rest of the chromatin was snap-frozen and stored at $-80^{\circ} \mathrm{C}$. Input DNA was treated with $10 \mu \mathrm{g}$ RNase A for $1 \mathrm{~h}$ at $37^{\circ} \mathrm{C}$ followed by a proteinase $\mathrm{K}$ treatment $(40 \mu \mathrm{g})$ for 1-2 h. DNA was extracted with phenol/chloroform (SigmaAldrich) and concentration was measured and used to calculate the total amount of chromatin in each sample.

For the pull-down, $20 \mu \mathrm{g}$ of chromatin was precleared with $10 \mu \mathrm{l}$ of Dynabeads protein G (Thermo Fisher Scientific), previously washed three times with RIPA $0.1 \%$ SDS (1× PBS, 1\% IGEPAL-C630, $0.5 \%$ sodium deoxycholate, $0.1 \%$ sodium dodecyl sulfate, and protease inhibitor cocktail [Roche]), for $2 \mathrm{~h}$ on the wheel at $4^{\circ} \mathrm{C} .1 / 10$ of the precleared chromatin was taken away and stored temporarily at $-20^{\circ} \mathrm{C}$, this was later used as the Input. The rest of the precleared chromatin was transferred into a new $1.5 \mathrm{ml}$ Eppendorf tube and incubated with $2 \mu \mathrm{g}$ of antibody for each condition overnight at $4^{\circ} \mathrm{C}$ (H3K9me3: ab8898, rabbit-IgG: NI01). $10 \mu$ lof Dynabeads protein G (Thermo Fisher Scientific) was added to the chromatin-antibody complexes and incubated $4 \mathrm{~h}$ on the wheel at $4^{\circ} \mathrm{C}$. Samples were placed on the magnetic rack and the supernatant was discarded. Samples were washed twice with wash buffer 1 (16.7 mM Tris-HCL, $\mathrm{pH} 8,0.167 \mathrm{M} \mathrm{NaCl}, 0.1 \%$ SDS, and $1 \%$ Triton $\mathrm{X}-100$ ) for 5 min rotating at room temperature. Then they were washed once with wash buffer 2 (16.7 mM Tris- $\mathrm{HCl}$, pH 8, 0.5M NaCl, 0.1\% SDS, and 1\% Triton $\mathrm{X}$-100) for $5 \mathrm{~min}$ rotating at room temperature and twice in LiCl wash buffer $(0.25 \mathrm{M} \mathrm{LiCl}, 0.5 \%$ sodium deoxycholate, $1 \mathrm{mM}$ EDTA, pH 8, $10 \mathrm{mM}$ Tris- $\mathrm{HCl}, \mathrm{pH}$ 8, and 0.5\% IGEPAL-CA630) for $5 \mathrm{~min}$ rotating at room temperature. Finally, the samples were washed twice in TE buffer (10 mM Tris- $\mathrm{HCl}, \mathrm{pH}$ 8, and 5 mM EDTA, pH 8) for 5 min rotating at room temperature. Samples were incubated in $300 \mu \mathrm{l}$ elution buffer ( $1 \% \mathrm{SDS}, 100 \mathrm{mM} \mathrm{NaHCO}$ ) for $30 \mathrm{~min}$ at $37^{\circ} \mathrm{C}$ shaking at 900 rpm (Eppendorf ThermoMixer C). Samples were placed on a magnetic rack and the supernatant transferred into a new Eppendorf tube containing $38.5 \mu \mathrm{l}$ Proteinase $\mathrm{K}$ mix $(15 \mu \mathrm{l} 1 \mathrm{M}$ Tris- $\mathrm{HCl}, \mathrm{pH} 8,15 \mu \mathrm{l} 5 \mathrm{M} \mathrm{NaCl}, 7.5 \mu \mathrm{l}$ 0.5M EDTA, pH 8, and $1 \mu \mathrm{l}$ Proteinase $\mathrm{K}[20 \mathrm{mg} / \mathrm{ml}]$ ). Also $300 \mu \mathrm{l}$ elution buffer and $38.5 \mu \mathrm{l}$ Proteinase $\mathrm{K}$ mix was added to the Inputs. Pull-downs and Inputs were incubated at $50^{\circ} \mathrm{C}$ for $3 \mathrm{~h}$ shaking at 1,100 rpm (Eppendorf 
ThermoMixer C) and then at $65^{\circ} \mathrm{C}$ overnight. DNA was treated with $10 \mu \mathrm{g}$ of RNase A for $45 \mathrm{~min}$ at $37^{\circ} \mathrm{C}$ and extracted with a phenol/ chloroform extraction, followed by an ethanol precipitation.

ChIPed and Input DNA was diluted 1:10 before the qPCR for the control Primers (Dazl, MusD and the intergenic region) and 1:50 before the qPCR for the major satellites. The qPCR was performed with the KAPA SYBR Fast qPCR Kit (Kapa Biosystems) and analyzed on a LightCycler 480 (Roche). The enrichment was calculated with the $2^{-\Delta \Delta C T}$ method over input. Control regions (Dazl, MusD [Karimi et al, 2011]) were represented as enrichment over the intergenic region (Ngondo et al, 2018). The enrichment for the major satellites was represented as the enrichment compared with WT. Primers are listed in Table S1.

\section{Major satellite computational analysis and binding site identification}

RepeatMasker annotations were obtained from UCSC for the mm10 mouse reference genome (http:/ / hgdownload.soe.ucsc.edu/goldenPath/ mm10/database/rmskOutCurrent.txt.gz) and filtered for major satellites (GSAT_MM) (Bao et al, 2015; Smit et al, 2013). Most regions annotated as major satellites were rather short ( $<1,000$ bps) and we only considered four regions, where the genome sequence contained $>20 \mathrm{Kbps}$ long major satellite regions. One of them was mapped into the $X$ chromosome and another one to chromosome 9. The other two fell into genomic contigs that could not be assigned to any chromosome (JH584304.1 and GL456383.1). For these four annotated regions, 8merbinding sites were scanned and counted for each mESC-expressed miRNA (Table S2). Small RNA-seq from WT mESCs has been previously analyzed in Schäfer et al (2021) Preprint and RIP-small RNA-seq in Ngondo et al (2018).

\section{miRNA inhibitor and mimic transfection}

Approximately 300,000-400,000 WT mESCs were plated the night before into a gelatin-coated $60.1 \mathrm{~cm}^{2}$ (B10) dish (TPP). The next day, for the miRNA inhibitor transfection, WT mESCs were either transfected with RNAiMax (Invitrogen) and $30 \mathrm{nM}$ of a negative control inhibitor (\#4464074; Ambion) or with RNAiMax (Invitrogen) and a mix of miR-30a-3p, miR-30d-3p, and miR-30e-3p inhibitors, $10 \mathrm{nM}$ for each inhibitor (\#4464084; Ambion). For the miRNA mimic transfection, WT mESCs were either transfected with RNAiMax (Invitrogen) and $30 \mathrm{nM}$ of a negative control mimic (CN-001000-0105; Dharmacon) or with RNAiMax (Invitrogen) and 30 nM of miR-30e3p mimics (C-310467-07-0002; Dharmacon). $36 \mathrm{~h}$ later, the cell pellet was collected. Briefly, cells were washed once with 1× PBS (Life Technologies), then trypsinized with $0.05 \%$ Trypsin-EDTA (Life Technologies) for $5 \mathrm{~min}$ at $37^{\circ} \mathrm{C}$. Trypsinization was stopped, by adding medium and spinning the cells down for $5 \mathrm{~min}$ at 182g. The cell pellet was washed once in $1 \times$ PBS (Life Technologies), spun down $5 \mathrm{~min}$ at $182 \mathrm{~g}$ and then stored at $-80^{\circ} \mathrm{C}$.

\section{Quantification and statistical analysis}

See Methods Details for details on quantification and statistical analysis. In general, statistical analysis was performed using PRIMS 8 as indicated in the figure legends.

\section{Data Availability}

Small RNA-seq (GSE80415) and RIP-small RNA-seq (GSE80454) (Ngondo et al, 2018) used in this study have been deposited in the Gene Expression Omnibus repository, analyzed results are provided in Table S2. References of images: FACS machine in Fig S3: https:// fluorofinder.com/cytometer-facsaria/. 96-well plate: https:// commons.wikimedia.org/wiki/File:96-Well_plate.svg.

\section{Supplementary Information}

Supplementary Information is available at https://doi.org/10.26508/lsa. 202101277.

\section{Acknowledgements}

We would like to thank the members of the Ciaudo Lab, Dr. Tobias Beyer, and Prof. Madhav Jagannathan (Swiss Federal Institute of Technology [ETH]) for fruitful discussions and the critical reading of this manuscript. We would also like to thank the Santoro lab for help with the ChIP protocol. This work was supported by the Swiss National Science Foundation (grants 31003A_173120 and 310030_196861) to C Ciaudo, R Arora and M Müller were supported by the National Center for Competence in Research (NCCR) RNA and disease. We are also thankful to the Scientific Centre for Optical and Electron Microscopy (ScopeM, ETH Zurich) for their support for imaging and the Flow Cytometry Core Facility of ETH for their help with FACS.

\section{Author Contributions}

M Mueller: conceptualization, formal analysis, supervision, validation, investigation, visualization, methodology, and writing-original draft, review, and editing.

T Faeh: conceptualization, formal analysis, validation, investigation, visualization, methodology, and writing-original draft, review, and editing. M Schaefer: data curation, software, visualization, methodology, and writing-review and editing.

$\checkmark$ Hermes: formal analysis, investigation, and visualization.

J Luitz: formal analysis and investigation.

P Stalder: formal analysis and investigation.

R Arora: supervision, investigation, methodology, and writing-review and editing.

RP Ngondo: formal analysis, supervision, investigation, and writing-review and editing.

C Ciaudo: conceptualization, resources, formal analysis, supervision, funding acquisition, visualization, methodology, project administration, and writing-original draft, review, and editing.

\section{Conflict of Interest Statement}

The authors declare that they have no conflict of interest.

\section{References}

Agirre E, Bellora N, Alló M, Pagès A, Bertucci P, Kornblihtt AR, Eyras E (2015) A chromatin code for alternative splicing involving a putative 
association between CTCF and HP1 $\alpha$ proteins. BMC Biol 13: 31. doi:10.1186/s12915-015-0141-5

Akhtar A, Gasser SM (2007) The nuclear envelope and transcriptional control. Nat Rev Genet 8: 507-517. doi:10.1038/nrg2122

Alisch RS, Jin P, Epstein M, Caspary T, Warren ST (2007) Argonaute2 is essential for mammalian gastrulation and proper mesoderm formation. PLoS Genet 3: e227. doi:10.1371/journal.pgen.0030227

Alló M, Agirre E, Bessonov S, Bertucci P, Gómez Acuña L, Buggiano V, Bellora N, Singh B, Petrillo E, Blaustein M, et al (2014) Argonaute-1 binds transcriptional enhancers and controls constitutive and alternative splicing in human cells. Proc Natl Acad Sci U S A 111: 15622-15629. doi:10.1073/pnas.1416858111

Ameyar-Zazoua M, Rachez C, Souidi M, Robin P, Fritsch L, Young R, Morozova N, Fenouil R, Descostes N, Andrau J-C, et al (2012) Argonaute proteins couple chromatin silencing to alternative splicing. Nat Struct Mol Biol 19: 998-1004. doi:10.1038/nsmb.2373

Bannister AJ, Zegerman P, Partridge JF, Miska EA, Thomas JO, Allshire RC, Kouzarides T (2001) Selective recognition of methylated lysine 9 on histone H3 by the HP1 chromo domain. Nature 410: 120-124. doi:10.1038/35065138

Bao W, Kojima KK, Kohany O (2015) Repbase update, a database of repetitive elements in eukaryotic genomes. Mob DNA 6: 11-19. doi:10.1186/ s13100-015-0041-9

Bartel DP (2018) Metazoan microRNAs. Cell 173: 20-51. doi:10.1016/ j.cell.2018.03.006

Bernard P, Maure JF, Partridge JF, Genier S, Javerzat JP, Allshire RC (2001) Requirement of heterochromatin for cohesion at centromeres. Science 294: 2539-2542. doi:10.1126/science.1064027

Bodak M, Ciaudo C (2016) Monitoring long interspersed nuclear element 1 expression during mouse embryonic stem cell differentiation. Methods Mol Biol 1400: 237-259. doi:10.1007/978-1-4939-3372-3_16

Bodak M, Cirera-Salinas D, Luitz J, Ciaudo C (2017) The role of RNA interference in stem cell biology: Beyond the mutant phenotypes. I Mol Biol 429: 1532-1543. doi:10.1016/j.jmb.2017.01.014

Boroviak T, Stirparo GG, Dietmann S, Hernando-Herraez I, Mohammed H, Reik W, Smith A, Sasaki E, Nichols J, Bertone P (2018) Single cell transcriptome analysis of human, marmoset and mouse embryos reveals common and divergent features of preimplantation development. Development 145: dev167833. doi:10.1242/dev.167833

Bühler M, Verdel A, Moazed D (2006) Tethering RITS to a nascent transcript initiates RNAi- and heterochromatin-dependent gene silencing. Cell 125: 873-886. doi:10.1016/j.cell.2006.04.025

Cheloufi S, Dos Santos CO, Chong MWM, Hannon GJ (2010) A dicerindependent miRNA biogenesis pathway that requires Ago catalysis. Nature 465: 584-589. doi:10.1038/nature09092

Cho S, Park JS, Kang YK (2014) AGO2 and SETDB1 cooperate in promotertargeted transcriptional silencing of the androgen receptor gene. Nucleic Acids Res 42: 13545-13556. doi:10.1093/nar/gku788

Cirera-Salinas D, Yu J, Bodak M, Ngondo RP, Herbert KM, Ciaudo C (2017) Noncanonical function of DGCR8 controls mESC exit from pluripotency. J Cell Biol 216: 355-366. doi:10.1083/jcb.201606073

DeVeale B, Swindlehurst-Chan J, Blelloch R (2021) The roles of microRNAs in mouse development. Nat Rev Genet 22: 307-323. doi:10.1038/s41576020-00309-5

Fallatah B, Shuaib M, Adroub S, Paytuví-Gallart A, Della Valle F, Nadeef S, Lanzuolo C, Orlando V (2021) Ago1 controls myogenic differentiation by regulating eRNA-mediated CBP-guided epigenome reprogramming. Cell Rep 37: 110066. doi:10.1016/j.celrep.2021.110066

Gagnon KT, Li L, Chu Y, Janowski BA, Corey DR (2014a) RNAi factors are present and active in human cell nuclei. Cell Rep 6: 211-221. doi:10.1016/ j.celrep.2013.12.013
Gagnon KT, Li L, Janowski BA, Corey DR (2014b) Analysis of nuclear RNA interference in human cells by subcellular fractionation and Argonaute loading. Nat Protoc 9: 2045-2060. doi:10.1038/ nprot.2014.135

Gao M, Wei W, Li MM, Wu YS, Ba Z, Jin KX, Li MM, Liao YQ, Adhikari S, Chong Z, et al (2014) Ago2 facilitates Rad51 recruitment and DNA double-strand break repair by homologous recombination. Cell Res 24: 532-541. doi:10.1038/cr.2014.36

Goto DB, Nakayama J (2012) RNA and epigenetic silencing: Insight from fission yeast. Dev Growth Differ 54: 129-141. doi:10.1111/j.1440169X.2011.01310.X

Guenatri M, Bailly D, Maison C, Almouzni G (2004) Mouse centric and pericentric satellite repeats form distinct functional heterochromatin. J Cell Biol 166: 493-505. doi:10.1083/jcb.200403109

Hall LE, Mitchell SE, O'Neill RJ (2012) Pericentric and centromeric transcription: A perfect balance required. Chromosome Res 20: 535-546. doi:10.1007/s10577-012-9297-9

Houlard M, Berlivet S, Probst AV, Quivy JP, Héry P, Almouzni G, Gérard M (2006) CAF-1 is essential for heterochromatin organization in pluripotent embryonic cells. PLoS Genet 2: e181. doi:10.1371/journal.pgen.0020181

Hsieh CL, Lin CL, Liu H, Chang YJ, Shih CJ, Zhong CZ, Lee SC, Tan BC (2011) WDHD1 modulates the post-transcriptional step of the centromeric silencing pathway. Nucleic Acids Res 39: 4048-4062. doi:10.1093/nar/ gkq1338

Hu J, Chen Z, Xia D, Wu J, Xu H, Ye ZQ (2012) Promoter-associated small double-stranded RNA interacts with heterogeneous nuclear ribonucleoprotein $\mathrm{A} 2 / \mathrm{B} 1$ to induce transcriptional activation. Biochem J 447: 407-416. doi:10.1042/BJ20120256

Huang V, Zheng J, Qi Z, Wang J, Place RF, Yu J, Li H, Li LC (2013) Ago1 interacts with RNA polymerase II and binds to the promoters of actively transcribed genes in human cancer cells. PLoS Genet 9: e1003821. doi:10.1371/journal.pgen.1003821

Hyun K, Jeon J, Park K, Kim J (2017) Writing, erasing and reading histone lysine methylations. Exp Mol Med 49: e324. doi:10.1038/emm.2017.11

Janowski BA, Huffman KE, Schwartz JC, Ram R, Nordsell R, Shames DS, Minna JD, Corey DR (2006) Involvement of AGO1 and AGO2 in mammalian transcriptional silencing. Nat Struct Mol Biol 13: 787-792. doi:10.1038/ nsmb1140

Kanellopoulou C, Muljo SA, Kung AL, Ganesan S, Drapkin R, Jenuwein T, Livingston DM, Rajewsky K (2005) Dicer-deficient mouse embryonic stem cells are defective in differentiation and centromeric silencing. Genes Dev 19: 489-501. doi:10.1101/gad.1248505

Karimi MM, Goyal P, Maksakova IA, Bilenky M, Leung D, Tang JX, Shinkai Y, Mager DL, Jones S, Hirst M, et al (2011) DNA methylation and SETDB1/ H3K9me3 regulate predominantly distinct sets of genes, retroelements, and chimeric transcripts in mESCs. Cell Stem Cell 8: 676-687. doi:10.1016/j.stem.2011.04.004

Kelly TJ, Brümmer A, Hooshdaran N, Tariveranmoshabad M, Zamudio JR (2019) Temporal control of the TGF- $\beta$ signaling network by mouse ESC MicroRNA targets of different affinities. Cell Rep 29: 2702-2717.e7. doi:10.1016/j.celrep.2019.10.109

Kim DH, Villeneuve LM, Morris KV, Rossi JJ (2006) Argonaute-1 directs siRNAmediated transcriptional gene silencing in human cells. Nat Struct Mol Biol 13: 793-797. doi:10.1038/nsmb1142

Komissarov AS, Gavrilova EV, Demin SJ, Ishov AM, Podgornaya OI (2011) Tandemly repeated DNA families in the mouse genome. BMC Genomics 12: 531. doi:10.1186/1471-2164-12-531

Lachner M, O'Carroll D, Rea S, Mechtler K, Jenuwein T (2001) Methylation of histone $\mathrm{H} 3$ lysine 9 creates a binding site for HP1 proteins. Nature 410: 116-120. doi:10.1038/35065132

Lehnertz B, Ueda Y, Derijck AA, Braunschweig U, Perez-Burgos L, Kubicek S, Chen T, Li E, Jenuwein T, Peters AH (2003) Suv39h-mediated histone H3 
lysine 9 methylation directs DNA methylation to major satellite repeats at pericentric heterochromatin. Curr Biol 13: 1192-1200. doi:10.1016/s0960-9822(03)00432-9

Li LC, Okino ST, Zhao H, Pookot D, Place RF, Urakami S, Enokida H, Dahiya R (2006) Small dsRNAs induce transcriptional activation in human cells. Proc Natl Acad Sci U S A 103: 17337-17342. doi:10.1073/pnas.0607015103

Li X, Wang X, Cheng Z, Zhu Q (2020) AGO2 and its partners: A silencing complex, a chromatin modulator, and new features. Crit Rev Biochem Mol Biol 55: 33-53. doi:10.1080/10409238.2020.1738331

Liu J, Carmell MA, Rivas FV, Marsden CG, Thomson JM, Song JJ, Hammond SM, Joshua-Tor L, Hannon GJ (2004) Argonaute2 is the catalytic engine of mammalian RNAi. Science 305: 1437-1441. doi:10.1126/science.1102513

Lykke-Andersen K, Gilchrist MJ, Grabarek JB, Das P, Miska E, Zernicka-Goetz M (2008) Maternal Argonaute 2 is essential for early mouse development at the maternal-zygotic transition. Mol Biol Cell 19: 4383-4392. doi:10.1091/mbc.e08-02-0219

Mcquin C, Goodman A, Chernyshev V, Kamentsky L, Cimini BA, Karhohs KW, Doan M, Ding L, Rafelski SM, Thirstrup D, et al (2018) Cellprofiler 3.0: Next-generation image processing for biology. PLoS Biol 16: e2005970. doi:10.1371/journal.pbio.2005970

Meister G (2013) Argonaute proteins: Functional insights and emerging roles. Nat Rev Genet 14: 447-459. doi:10.1038/nrg3462

Modzelewski AJ, Holmes RJ, Hilz S, Grimson A, Cohen PE (2012) AGO4 regulates entry into meiosis and influences silencing of sex chromosomes in the male mouse germline. Dev Cell 23: 251-264. doi:10.1016/ j.devcel.2012.07.003

Morita S, Horii T, Kimura M, Goto Y, Ochiya T, Hatada I (2007) One Argonaute family member, Eif2c2 (Ago2), is essential for development and appears not to be involved in DNA methylation. Genomics 89: 687-696. doi:10.1016/j.ygeno.2007.01.004

Motamedi MR, Verdel A, Colmenares SU, Gerber SA, Gygi SP, Moazed D (2004) Two RNAi complexes, RITS and RDRC, physically interact and localize to noncoding centromeric RNAs. Cell 119: 789-802. doi:10.1016/ j.cell.2004.11.034

Mueller M, Schaefer M, Faeh T, Spies D, Peña-Hernández R, Santoro R, Ciaudo C (2021) ARGONAUTE proteins regulate a specific network of genes through KLF4 in mouse embryonic stem cells. BioRxiv. doi:10.1101/ 2021.10.18.464771. (Preprint posted October 19, 2021)

Müller M, Fazi F, Ciaudo C (2020) Argonaute proteins: From structure to function in development and pathological cell fate determination. Front Cell Dev Biol 7: 360. doi:10.3389/fcell.2019.00360

Ngondo RP, Cirera-Salinas D, Yu J, Wischnewski H, Bodak M, VandormaelPournin S, Geiselmann A, Wettstein R, Luitz J, Cohen-Tannoudji M, et al (2018) Argonaute 2 is required for extra-embryonic endoderm differentiation of mouse embryonic stem cells. Stem Cell Rep 10: 461-476. doi:10.1016/j.stemcr.2017.12.023

Nishi K, Nishi A, Nagasawa T, Ui-Tei K (2013) Human TNRC6A is an Argonautenavigator protein for microRNA-mediated gene silencing in the nucleus. RNA 19: 17-35. doi:10.1261/rna.034769.112

Nonaka N, Kitajima T, Yokobayashi S, Xiao G, Yamamoto M, Grewal SI, Watanabe $Y$ (2002) Recruitment of cohesin to heterochromatic regions by Swi6/HP1 in fission yeast. Nat Cell Biol 4: 89-93. doi:10.1038/ncb739

Portnoy V, Lin SH, Li KH, Burlingame A, Hu ZH, Li H, Li LC (2016) SaRNA-guided Ago2 targets the RITA complex to promoters to stimulate transcription. Cell Res 26: 320-335. doi:10.1038/cr.2016.22

Probst AV, Almouzni G (2008) Pericentric heterochromatin: Dynamic organization during early development in mammals. Differentiation 76: 15-23. doi:10.1111/j.1432-0436.2007.00220.x

Probst AV, Dunleavy E, Almouzni G (2009) Epigenetic inheritance during the cell cycle. Nat Rev Mol Cell Biol 10: 192-206. doi:10.1038/nrm2640
Probst AV, Okamoto I, Casanova M, El Marjou F, Le Baccon P, Almouzni G (2010) A strand-specific burst in transcription of pericentric satellites is required for chromocenter formation and early mouse development. Dev Cell 19: 625-638. doi:10.1016/j.devcel.2010.09.002

Rudert F, Bronner S, Garnier JM, Dollé P (1995) Transcripts from opposite strands of gamma satellite DNA are differentially expressed during mouse development. Mamm Genome 6: 76-83. doi:10.1007/BF00303248

Sarshad AA, Juan AH, Muler AIC, Anastasakis DG, Wang X, Genzor P, Feng X, Tsai PF, Sun HW, Haase AD, et al (2018) Argonaute-miRNA complexes silence target mRNAs in the nucleus of mammalian stem cells. Mol Cell 71: 1040-1050.e8. doi:10.1016/j.molcel.2018.07.020

Schäfer M, Nabih A, Spies D, Bodak M, Stalder P, Ngondo RP, Liechti LA, Sajic T, Aebersold R, Gatfield D, et al (2021) Integrative analysis allows a global and precise identification of functional miRNA target genes in mESCS. BioRxiv. doi:10.1101/2021.09.24.461622. (Preprint posted September 29, 2021)

Schindelin J, Arganda-Carreras I, Frise E, Kaynig V, Longair M, Pietzsch T, Preibisch S, Rueden C, Saalfeld S, Schmid B, et al (2012) Fiji: An opensource platform for biological-image analysis. Nat Methods 9: 676-682. doi:10.1038/nmeth.2019

Shivram H, Le SV, Iyer VR (2019) MicroRNAs reinforce repression of PRC2 transcriptional targets independently and through a feed-forward regulatory network. Genome Res 29: 184-192. doi:10.1101/gr.238311.118

Shuaib M, Parsi KM, Thimma M, Adroub SA, Kawaji H, Seridi L, Ghosheh Y, Fort A, Fallatah B, Ravasi T, et al (2019) Nuclear AG01 regulates gene expression by affecting chromatin architecture in human cells. Cell Syst 9: 446-458.e6. doi:10.1016/j.cels.2019.09.005

Smit A, Hubley R, Green P (2013) RepeatMasker Open-4.0. www.repeatmasker.org.

Solovei I, Kreysing M, Lanctôt C, Kösem S, Peichl L, Cremer T, Guck J, Joffe B (2009) Nuclear architecture of rod photoreceptor cells adapts to vision in mammalian evolution. Cell 137: 356-368. doi:10.1016/j.cell.2009.01.052

Storer J, Hubley R, Rosen J, Wheeler TJ, Smit AF (2021) The Dfam community resource of transposable element families, sequence models, and genome annotations. Mob DNA 12: 2-14. doi:10.1186/s13100-020-00230-y

Van Stry M, Oguin TH, Cheloufi S, Vogel P, Watanabe M, Pillai MR, Dash P, Thomas PG, Hannon GJ, Bix M (2012) Enhanced susceptibility of Ago1/3 double-null mice to influenza A virus infection. J Virol 86: 4151-4157. doi:10.1128/JVI.05303-11

Verdel A, Jia S, Gerber S, Sugiyama T, Gygi S, Grewal SI, Moazed D (2004) RNAimediated targeting of heterochromatin by the RITS complex. Science 303: 672-676. doi:10.1126/science.1093686

Vissel B, Choo KH (1989) Mouse major (gamma) satellite DNA is highly conserved and organized into extremely long tandem arrays: Implications for recombination between nonhomologous chromosomes. Genomics 5 : 407-414. doi:10.1016/0888-7543(89)90003-7

Wang Q, Goldstein M (2016) Small RNAs recruit chromatin-modifying enzymes MMSET and Tip60 to reconfigure damaged DNA upon double-strand break and facilitate repair. Cancer Res 76: 1904-1915. doi:10.1158/0008-5472.CAN-15-2334

Wettstein R, Bodak M, Ciaudo C (2016) Generation of a knockout mouse embryonic stem cell line using a paired CRISPR/Cas9 genome engineering tool. Methods Mol Biol 1341: 321-343. doi:10.1007/ 7651_2015_213

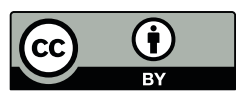

License: This article is available under a Creative Commons License (Attribution 4.0 International, as described at https://creativecommons.org/ licenses/by/4.0/). 